\title{
PWR Blowdown Heat Transfer Separate-Effects Program-Thermal-Hydraulic Test Facility Experimental Data Report for Test 103
}

\author{
V. D. Clemons \\ M. D. White \\ P. A. Moore \\ R. A. Hedrick
}

Prepared for the U.S. Nuc!ear Regulatory Commission Office of Nuclear Regulatory Research

Under Interagency f.greements DOE 40-551-75 and 40-552-75

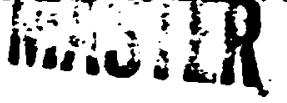


ORNL/NUREG/TM-187

Dist - Category NRC-2

Contract No. W-7405-eng-26

Èngineering Technology Division

PWR BLOWDOWN HEAT TRANSFER SEPARATE-EFFECTS

PROGRAM - THERMAL-HYDRAULIC TEST FACILITY

EXPERIMENTAL DATA REPORT FOR TEST 103

V. D. Clemons

P. A. Moore

M. D. White

R. A. Hedrick

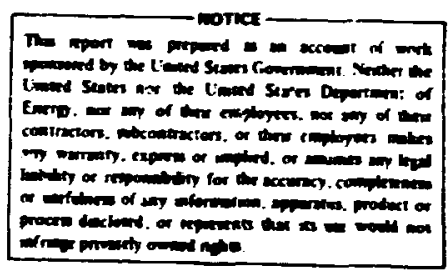

Manuscript Completed - March 7, 1978

Date Published - March 1978

NOTICE: This document contains information of a preliminary natire. It is subject to revision or correction and therefore does not represent a final report.

\author{
Prepared for the \\ U.S. Nuclear Regulatory Commission \\ office of Nuclear Regulatory Research \\ Under Interagency Agreements DOF, 40-551-75 and 40-552-75
}

Prepared by the

OAK RIDGE NATINNAL LABORATORY

Oak Ridge, Tennessee $378 j 0$

operated by

UNION CARBIDE CORPORATION

for the

DEPARTMENT OF ENERGY 
ACKAONLEDGMENTS $\ldots \ldots \ldots \ldots \ldots \ldots \ldots \ldots \ldots \ldots \ldots \ldots \ldots \ldots \ldots \ldots \ldots \ldots \ldots$

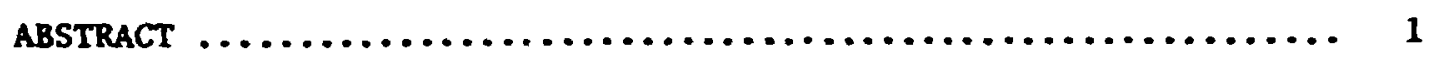

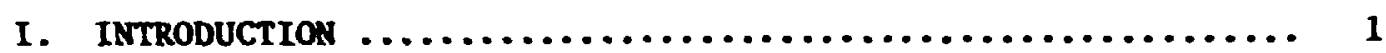

II. SYSTEM, PROCEDURES, CONDITIONS, AND EVEATS FOR TEST $104 \ldots \ldots$

1. System Configuration and Test Procedure ............. 2

2. Initial Test Conditions and Sequence of Events ........ 4

III. DATA PRESEMTATION $\ldots \ldots \ldots \ldots \ldots \ldots \ldots \ldots \ldots \ldots \ldots \ldots \ldots \ldots \ldots$

\section{TABLES}

1. Desired vs actual prerupture conditions ............... 4

2. Prerupture primary-coolant temperature and pressure distribution - test $103 \ldots \ldots \ldots \ldots \ldots \ldots \ldots \ldots \ldots \ldots \ldots \ldots$

3. Sequence of events during test $103 \ldots \ldots \ldots \ldots \ldots \ldots \ldots \ldots$

4. Precision of experimental measurements for test $103 \ldots \ldots . .7$

5. Data presentation for test $103 \ldots \ldots \ldots \ldots \ldots \ldots \ldots \ldots \ldots \ldots$

\section{FIGURES}

1. Thermal-Hydraulic Test Facility (THTF) $\ldots \ldots \ldots \ldots \ldots \ldots \ldots \ldots$

2. Identification of THTF heater rod and subchannel locations in bundles 1 and $2 \ldots \ldots \ldots \ldots \ldots \ldots \ldots \ldots \ldots . \ldots \ldots$

3. Locat fon of thermocouples in THTF bundle $1 \ldots \ldots \ldots \ldots \ldots \ldots$

4. THTF instrument identification and location $\ldots \ldots \ldots \ldots \ldots . .8$

[Figures 5 through 504 have no page numbers assigned in microfiche edition of this report.]

5. TE-301AD, sheath thermocouple, rod 1 , level $D \ldots \ldots \ldots \ldots \ldots 28$

6. TE-304AD, sheath thermocouple, rod 4 , level $\mathrm{D} \ldots \ldots \ldots \ldots . .28$

7. TE-309AD, sheath thermocouple, rod 9 , level D .......... 29

8. TE-310AD, sheath thermocouple, $\operatorname{rod} 10$, level $D \ldots \ldots \ldots \ldots . .29$

9. TE-312AD, sheath thermocouple, rod 12, level $D \ldots \ldots \ldots \ldots \ldots$

10. TE-313AD, sheath thermocouple, rod 13, level D ......... 30

11. TE-317AD, sheath thermocouple, $\operatorname{rod} 17$, level $D \ldots \ldots \ldots \ldots . .31$

12. TE-318AD, sheath thermocouple, $\operatorname{rod} 18$, level D ........ 31

13. TE-320AD, sheath thermocouple, $\operatorname{rod} 20$, leve1 D ........ 32

14. TE-322AD, sheath thermocouple, $\operatorname{rod} 22$, leve1 $\mathrm{D} \ldots \ldots \ldots \ldots . \ldots 32$ 
BLANK PAGE 
15. TE-323AD, sheath thernocouple, $\operatorname{rod} 23$, level $D \ldots \ldots \ldots \ldots \ldots 33$

16. TE-325AD, sheath thermocouple, $\operatorname{rod} 25$, level D .......... 33

17. TE-326AD, sheath thernocouple, rod 26, level D .......... 34

18. TE-331AD, sheath thermocouple, rod 31, level $D \ldots \ldots \ldots \ldots \ldots . . .34$

19. TE-338AD, sheath thernocouple, rod 38, level D .......... 35

20. TE-339AD, sheath thermocouple, rod 39, level D .......... 35

21. TE-341AD, sheath thernocouple, $\operatorname{rod} 41$, level D ......... 36

22. TE-349AD, sheath thernocouple, $\operatorname{rod} 49$, level $D \ldots \ldots \ldots \ldots \ldots 36$

23. TE-301AE, sheath therwocouple, $\operatorname{rod} 1$, level E .......... 37

24. TE-304AE, sheath thermocouple, rod 4, level E .......... 37

25. TE-309AE, sheath thermocouple, $\operatorname{rod} 9$, level E .......... 38

26. TE-312AE, sheath thermocouple, $\operatorname{rod} 12$, level $E \ldots \ldots \ldots \ldots \ldots 38$

27. TE-313AE, sheath thermocouple, rod 13, level E .......... 39

28. TE-317AE, sheath thermocouple, $\operatorname{rod} 17$, level E ......... 39

29. TE-318AE, sheath thermocouple, $\operatorname{rod} 18$, level $E \ldots \ldots \ldots \ldots$ i0

30. TH-320AE, sheath thermocouple, $\operatorname{rod} 20$, level E $\ldots \ldots \ldots \ldots \ldots .40$

31. TE-322AE, sheath therwocouple, $\operatorname{rod} 22$, level E $\ldots \ldots \ldots \ldots \ldots .41$

32. TE-323AE, sheath therwocouple, $\operatorname{rod} 23$, level E $\ldots \ldots \ldots \ldots \ldots 41$

33. TE-324AE, sheath thermocouple, rod 24, level E ......... 42

34. TE-325AE, sheath thermocouple, $\operatorname{rod} 25$, level E ......... 42

35. TE-326AE, sheath thernocouple, rod 26 , level E ........ 43

36. TE-331AE, sheath thermocouple, $\operatorname{rod} 31$, level $E \ldots \ldots \ldots \ldots . .43$

37. TE-333AE, sheath thernocouple, rod 33, level E ......... 44

38. TE-338AE, sheath thernocouple, $\operatorname{rod} 38$, level E .......... 44

39. TE-339AE, sheath thernocouple, $\operatorname{rod} 39$, level $E \ldots \ldots \ldots \ldots .45$

40. TE-341AE, sheath thernocouple, $\operatorname{rod} 41$, level E ......... 45

41. TE-349AE, sheath therecouple, rod 49, level E .......... 40́

42. TE-301BF, sheath thermocouple, rod 1, level $\mathrm{F} \ldots \ldots \ldots \ldots \ldots 46$

43. TE-304BF, sheath thernocouple, rod 4 , level $\mathbf{F} \ldots \ldots \ldots \ldots . .47$

44. TE-309BF, sheath thermocouple, $\operatorname{rod} 9$, level $F \ldots \ldots \ldots \ldots \ldots 4$

45. TE-310BF, sheath thermocouple, rod 10 , level $F \ldots \ldots \ldots \ldots . .48$

46. TE-312BF, sheath therwocouple, rod 12, level $F \ldots \ldots \ldots \ldots \ldots 48$

47. TE-3138F, sheath therwocouple, rod 13, ievel $F \ldots \ldots \ldots \ldots . .49$

48. TE-317BF, sheath thermocouple, $\operatorname{rod} 17$, level $F \ldots \ldots \ldots \ldots . .49$

49. TE-320BF, sheath therwocouple, rod 20, level $F \ldots \ldots \ldots \ldots . .50$ 
Page

50. TE-322BF, sheath thernocouple, rod 22 , level $F \ldots \ldots \ldots \ldots \ldots$ s0

51. TE-323BF, sheath therwocouple, $\operatorname{rod} 23$, level $F \ldots \ldots \ldots \ldots . .51$

52. TE-324BF, sheath thernocouple, $\operatorname{rod} 24$, level $F \ldots \ldots \ldots \ldots . . .51$

53. TE-325BF, sheath therwocouple, $\operatorname{rod} 25$, level $F \ldots \ldots \ldots \ldots .52$

54. TE-326BF, sheath therwecouple, rod 26, level F ......... 52

55. TE-331BF, sheath thermocouple, $\operatorname{rod} 31$, level $F \ldots \ldots \ldots \ldots .53$

56. TE-353BF, sheach therecouple, $\operatorname{rod} 33$, level $F \ldots \ldots \ldots \ldots .53$

57. TE-338BF, sheath thernocouple, $\operatorname{rod} 38$, level $F \ldots \ldots \ldots \ldots .54$

58. TE-341BF, sheach thermocouple, $\operatorname{rod} 41$, level $F \ldots \ldots \ldots \ldots .54$

59. TE-349BF, sheach thermocouple, $\operatorname{rod} 4^{r}$, level $F \ldots \ldots \ldots \ldots$ s5

60. TE-301BG, sheath thereocouple, rod 1, level $G \ldots \ldots \ldots \ldots \ldots$ s5

61. TE-304BG, sheath therwocouple, $\operatorname{rod} 4$, level $G \ldots \ldots \ldots \ldots \ldots .56$

62. TE-309BG, sheath thernocouple, $\operatorname{rod} 9$, level $G \ldots \ldots \ldots \ldots . .56$

63. TE-310BG, sheath thernocouple, $\operatorname{rod} 10$, level $G \ldots \ldots \ldots \ldots \ldots 57$

64, TE-312BG, sheach therwocouple, $\operatorname{rod} 12$, level $G \ldots \ldots \ldots \ldots \ldots 57$

65. TE-313BG, sheath thernoccuple, $\operatorname{rod} 13$, level $G \ldots \ldots \ldots \ldots .58$

66. TE-317BG, sheath thermocouple, $\operatorname{rod} 17$, level $G \ldots \ldots \ldots \ldots .58$

67. TE-318BC, sheath therwocouple, $\operatorname{rod} 18$, level $G \ldots \ldots \ldots \ldots .59$

68. TE-320BG, sheath therwocouple, $\operatorname{rod} 20$, level $G \ldots \ldots \ldots \ldots . .59$

69. TE-322BG, sheath therwocouple, $\operatorname{rod} 22$, level G ......... 60

70. TE-323BG, sheach thermocouple, $\operatorname{rod} 23$, level $G \ldots \ldots \ldots \ldots .60$

71. TE-325BG, sheath thermocouple, $\operatorname{rod} 25$, level $G \ldots \ldots \ldots \ldots .61$

72. TE-326BG, sheath thermocouple, $\operatorname{rod} 26$, level G ......... 61

73. TE-331BG, sheath thermocouple, $\operatorname{rod} 31$, level G ......... 62

74. TE-333BG, sheath thermocouple, $\operatorname{rod} 33$, level G ......... 62

75. TE-338BG, sheath thermocouple, $\operatorname{rod} 38$, level G ......... 63

76. TE-339BG, sheath thermocouple, rod 39, level G ......... 63

77. TE-341BG, sheath thermocouple, $\operatorname{rod} 41$, level G .......... 64

78. TE-349BG, sheath thermocouple, $\operatorname{rod} 49$, level G .......... 64

79. TE-301CH, sheath thermocouple, $\operatorname{rod} 1$, level H ........... 65

80. TE-302AH, sheath thermocouple, rod 2 , level H .......... 65

81. TE-303AH, sheath thermocouple, rod 3, level H .......... 66

82. TE-304CH, sheath thermocouple, rot 4, level H .......... 66

83. TE-305AH, sheath thermocouple, rod 5 , level н ........... 67 
Page

84. TE-306AH, sheath chernocouple, rod 6, level H .......... 67

85. TE-307AH, sheath thermocouple, $\operatorname{rod} 7$, level H .......... 68

86. TE-308AH, shea:h thermocouple, $\operatorname{rod} 8$, level H .......... 68

87. TE-309cu, sheath thermocouple, rod 9, level H.......... 69

88. TE-310CH, sheath thermocouple, $\operatorname{rod} 10$, level H .......... 69

89. TE-311AH, sheath thermocouple, rod 11, level H ......... 70

90. TE-312CH, sheath thermocouple, $\operatorname{rod} 12$, level H .......... 70

91. TE-313CH, sheath thermocouple, $\operatorname{rod} 13$, level H ......... 71

92. TE-314AH, sheath thermocouple, $\operatorname{rod} 14$, level H $\ldots \ldots \ldots \ldots .71$

93. TE-315All, sheath thermocouple, $\operatorname{rod} 15$, level H $\ldots \ldots \ldots \ldots .72$

94. TE-316AH, sheath thermocouple, $\operatorname{rod} 16$, level H $\ldots \ldots \ldots \ldots . .72$

95. TE-317CH, sheath thernocouple, $\operatorname{rod} 17$, level $\mathrm{H} \ldots \ldots \ldots \ldots .73$

96. TE-318CH, sheath thermocouple, $\operatorname{rod} 18$, level H $\ldots \ldots \ldots \ldots .73$

97. TE-320CH, sheath thernocouple, rod 20, level H ......... 74

98. TE-321AH, sheath thernocouple, $\operatorname{rod} 21$, level H ......... 74

99. TE-322CH, sheath thermocouple, $\operatorname{rod} 22$, level H ........ 75

100. TE-323СН, sheath thernocouple, $\operatorname{rod} 23$, level H ......... 75

101. TE-324CH, sheath thernocouple, rod 24 , level H ........ 76

102. TE-325Ch, sheath thermocouple, $\operatorname{rod} 25$, level H .......... 76

103. TE-326CH, sheath thermocouple, $\operatorname{rod} 26$, level H ......... 77

104. TE-327AH, sheath therwocouple, $\operatorname{rod} 27$, level H ......... 77

105. TE-328AH, sheath thermocouple, rod 28, level H ........ 78

106. TE-331CH, sheath thermocouple, rod 31, level H ......... 78

107. TE-333CH, sheath thermocouple, $\operatorname{rod} 33$, level H ......... 79

108. TE-336AH, sheath thermocouple, rod 36, level hi........ 79

109. TE-337AH, sheath thermocouple, rod 3i, level H ......... 80

110. TE-338CH, sheath therwocouple, rod 38 , level H ......... 80

111. TE-339CH, sheath thermocouple, rod 39 , level H........ 81

112. TE-340AH, sheath thermocouple, rod 40, level H........ 81

113. TE-341CH, sheath thermocouple, rod 41, level H......... 82

114. TE. 342AH, sheath thermocouple, rod 42 , level H ........ 82

115. TE-343AH, sheath thermocouple, $\operatorname{rod} 43$, level H ......... 83

116. TE-344AH, sheath thermocouple, rod 44, level H ......... 83

117. TE-345AH, sheath thermocouple, rod 45 , level H ......... 84 
118. TE-346AH, sheath therwocouple, $\operatorname{rod} 46$, level H $\ldots \ldots \ldots \ldots \ldots 84$

119. TE-348AH, sheath therwocouple, $\operatorname{rod} 48$, level H .......... 85

120. TE-349CH, sheath thermocouple, $\operatorname{rod} 49$, level H .......... 85

121. TE-301CI, sheath thernocouple, $\operatorname{rod} 1$, level I .......... 86

122. TE-302AI, sieath thernocouple, rod 2 , level I .......... 86

123. TE-303AI, sheath thermocouple, $\operatorname{rod} 3$, level I $\ldots \ldots \ldots \ldots \ldots .87$

124. TE-304CI, sheath thernocouple, $\operatorname{rod} 4$, level I $\ldots \ldots \ldots \ldots \ldots .87$

125. TE-305AI, sheath thermocouple, rod 5, level I ........... 88

126. TE-306AI, sheath thermocouple, rod 6, level I .......... 88

127. TE-307AI, sheath thernocouple, $\operatorname{rod} 7$, level I .......... 89

128. TE-308AI, sheath therwocouple, $\operatorname{rod} 8$, level I .......... 89

129. TE-309CI, sheath thermocouple, $\operatorname{rod} 9$, level I .......... 90

130. TE-310CI, sheath therwocoupie, $\operatorname{rod} 10$, level I ......... 90

131. TE-311AI, sheath thernocouple, rod 11 , level I ......... 91

132. TE-312CI, sheath thernocouple, roo 12, level I ......... 91

133. Tr-313CI, sheath thereocouple, rod 13, level I ......... 92

134. TE-314AI, sheath therwocouple, rod 14, level I ......... 92

135. TE-315AI, sheath thermccouple, rod 15, level I ......... 93

136. TE-316AI, sheath therwocouple, rod 16, level I ......... 93

137. TE-317CI, sheath therwocouple, $\operatorname{rod} 17$, level I .......... 94

138. TE-318CI, sheath thermocouple, rod 18, level I ......... 94

139. TE-320CI, sheath thermocouple, rod 20, level I ......... 95

140. TE-321AI, sheath thermocouple, rod 21 , level I ......... 95

141. TE-322CI, sheath thermocouple, $\operatorname{rod} 22$, level I ........ 96

142. TE-323CI, sheath thermocouple, rod 23, level I ......... 96

143. TE-324CI, sheath thermocouple, rod 24, level I .......... 97

144. TE-325CI, sheath thermocouple, $\operatorname{rod} 25$, level I ......... 97

145. TE-326CI, sheath thermocouple, rod 26, level I ......... 98

146. TE-327AI, sheath thermocouple, $\operatorname{rod} 27$, level I ........ 98

147. TE-328AI, sheath thernocouple, rod 28, level I ......... 99

148. TE-331CI, sheath thermscouple, rod 31 , level I .......... 99

149. TE-333CI, sheath thermocouple, $\operatorname{rod} 33$, level I ......... 100

150. TE-336AI, sheath thermocouple, rod 36, level I ......... 100

151. TE-337AI, sheath thermocouple, rod 37, level I .......... 101 
152. TE-338CI, sheath thernocouple, $\operatorname{rod} 38$, level I $\ldots \ldots \ldots \ldots \ldots 101$

153. TE-339CI, sheath chermocouple, rod 39, level I .......... 102

154. TE-340AI, sheath thernocouple, $\operatorname{rod} 40$, level I $\ldots \ldots \ldots \ldots \ldots 102$

155. TE-341CI, sheath thermocouple, $\operatorname{rod} 41$, level I .......... 103

156. TE-342AI, sheach thernocouple, $\operatorname{rod} 42$, level I ......... 103

157. TE-343AI, sheath thernocouple, $\operatorname{rod} 43$, level I ......... 104

158. TE-364AI, sheath thernocouple, rod 44 , level I ......... 104

159. TE-345AI, sheath thernosouple, $\operatorname{rod} 45$, level I ......... 105

160. TE-346AI, sheath thernocouple, rod 46, level I .......... 105

161. TE-348AI, sheach thernocouple, rod 48, level I .......... 106

162. TE-349CI, sheath thernocouple, rod 49, level I .......... 106

163. TE-301DJ, sheath thernocouple, rod 1, level J .......... 107

164. TE-302CJ, sheath thernocouple, rod 2, level J ........... 107

165. TE-303CJ, sheath thermocouple, $\operatorname{rod} 3$, level $\mathrm{J} \ldots \ldots \ldots \ldots \ldots . . .108$

166. TE-304DJ, sheath therwocouple, rod 4, level J .......... 108

167. TE-305CJ, sheath thermocouple, $\operatorname{rod} 5$, level $\mathrm{J} \ldots \ldots \ldots \ldots . .109$

168. TE-306CJ, sheath thermocouple, $\operatorname{rod} 6$, level J .......... 109

169. TE-307CJ, sheath thermocouple, $\operatorname{rod} 7$, level $\mathrm{J} \ldots \ldots \ldots \ldots \ldots 110$

170. TE-308CJ, sheath thermocouple, $\operatorname{rod} 8$, level $\mathrm{J} \ldots \ldots \ldots \ldots \ldots$

171. TE-309DJ, sheath thermocouple, rod 9, level J .......... 111

172. TE-310DJ, sheath thermocouple, rod 10, level $\mathrm{J} \ldots \ldots \ldots \ldots \ldots 11$

173. TE-311CJ, sheath thermocouple, rod 11, level $\mathrm{J} \ldots \ldots \ldots \ldots \ldots 112$

174. TE-312DJ, sheath therwocouple, rod 12, level J ......... 112

175. TE-313DJ, sheath therwocouple, $\operatorname{rod} 13$, level $\mathrm{J} \ldots \ldots \ldots \ldots 113$

176. TE-314CJ, sheath thernocouple, rod 14, level J ......... 113

177. TE-316CJ, sheath therwocouple, rod 16, level J ......... 114

178. TE-317DJ, sheath therwocouple, rod 17, level J .......... 114

179. TE-318DJ, sheath thermocouple, rod 18, level J ......... 115

180. TE-320DJ, sheath thermocouple, rod 20, level J ......... 115

181. TE-321CJ, sheath thermocouple, rod 21, level J ........ 116

182. TE-322DJ, sheath therwocouple, $\operatorname{rod} 22$, level $\mathrm{J} \ldots \ldots \ldots \ldots . .116$

183. TE-323DJ, sheath thermocouple, rod 23, level J ......... 117

184. TE-324D.J, sheath thermocouple, rod 24, level J ......... 117

185. TE-325DJ, sheath thermocouple, rod 25, level J ......... 118 
186. TE-326DJ, sheath thermocouple, $\operatorname{rod} 26$, level $\mathrm{J} \ldots \ldots \ldots \ldots \ldots 118$

187. TE-327CJ, sheath thernocouple, $\operatorname{rod} 27$, level $\mathrm{J} \ldots \ldots \ldots \ldots . .119$

188. TE-328CJ, sheath thernocouple, rod 28 , level J .......... 119

189. TE-331DJ, sheath therwocouple, rod 31, level J ......... 120

190. TE-333DJ, sheath thernocouple, $\operatorname{rod} 33$, level $J \ldots \ldots \ldots \ldots \ldots 120$

191. TE-336CJ, sheath thermocouple, rod 36, level J ......... 121

192. TE-337CJ, sheach thernocouple, rod 37, level J ......... 121

193. TE-338DJ, sheath thermocouple, rod 38, level J ........ Iz2

194. TE-339DJ, sheath thermocouple, rod 39, level J ......... 122

195. TE-340CJ, sheath thernocouple, rod 40, level $\mathrm{J} \ldots \ldots \ldots \ldots 123$

196. TE-341DJ, sheath thernocouple, rod 41, level $\mathrm{J} \ldots \ldots \ldots \ldots 123$

197. TE-342CJ, sheath thermocouple, $\operatorname{rod} 42$, level $\mathrm{J} \ldots \ldots \ldots \ldots \ldots 124$

198. TE-343CJ, sheath thermocouple, $\operatorname{rod} 43$, level $\mathrm{J} \ldots \ldots \ldots \ldots \ldots 124$

199. TE-344CJ, sheath thernocouple, rod 44, level $J \ldots \ldots \ldots \ldots . .125$

200. TE-345CJ, sheath thermocouple, $\operatorname{rod} 45$, level J ......... 125

201. TE-346CJ, sheach thermocouple, $\operatorname{rod} 46$, level J $\ldots \ldots \ldots \ldots .126$

202. TE-347CJ, sheath thermocouple, $\operatorname{rod} 47$, level J ......... 126

203. TE-349DJ, sheath thermocouple, rod 49, level J ......... 127

204. TE-301DK, sheath thermocouple, rod 1, level $K \ldots \ldots \ldots \ldots \ldots 127$

205. TE-304DK, sheath thernocouple, rod 4, level $K \ldots \ldots \ldots \ldots \ldots 128$

206. TE-309DK, sheath therwocouple, rod 9, level $K \ldots \ldots \ldots \ldots \ldots 128$

207. TE-310DK, sheath thermocouple, $\operatorname{rod} 10$, level $K \ldots \ldots . . . . .129$

208. TE-3120K, sheath thermocouple, $\operatorname{rod} 12$, level $\mathrm{K} \ldots \ldots \ldots \ldots . . .129$

209. TE-313DK, sheath thermocouple, rod 13, level K ........ 130

210. TE-317DK, sheath thermoccuple, rod 17, level K .......... 130

211. TE-318DK, sheath thermocouple, rod 18, level $K \ldots \ldots \ldots \ldots \ldots 131$

212. TE-320DK, sheath thermocouple, rod 20 , level $K \ldots \ldots \ldots \ldots \ldots 131$

213. TE-322DK, sheath thermocouple, rod 22 , level $K \ldots \ldots \ldots \ldots 132$

214. TE-323DK, sheath thermocouple, $\operatorname{rod} 23$, level $K \ldots \ldots \ldots \ldots 132$

215. TE-324DK, sheath thermocouple, rod 24 , level K .......... 133

216. TE-325DK, sheath thermocouple, rod 25 , level $K \ldots \ldots \ldots \ldots 133$

217. TE-3': गK, sheath thermocouple, rod 26 , level $K \ldots \ldots \ldots \ldots 134$

218. TE-331DK, sheath thermocouple, rod 31, level $K \ldots \ldots \ldots \ldots . .134$

213. TE-333DK, sheath thermocouple, rod 33, level $k \ldots \ldots \ldots \ldots 135$ 
Page

220. TE-3380k, sheath thernocouple, rod 38, level K......... 135

221. TE-339DK, sbeath thernocouple, rod 39, level K ......... 136

222. TE-341DK, sheath therwocouple, $\operatorname{rod} 41$, level $R \ldots \ldots \ldots \ldots \ldots 136$

223. TE-349DK, sheath therwocouple, rod 49, level $K \ldots \ldots \ldots \ldots \ldots 137$

224. TE-301EL, sheath thermocouple, $\operatorname{rod} 1$, level $L \ldots \ldots \ldots \ldots \ldots 137$

225. TE-302CL, sheath therwocouple, rod 2, level L ........... 138

226. TE-303CL, sheath thermocouple, rod 3, level L .......... 138

227. TE-304EL, sheath therwocouple, rod 4, level L ........... 139

228. TE-30SCL, sheath therwocouple, $\operatorname{rod} 5$, level $\mathrm{L} \ldots \ldots \ldots \ldots \ldots 139$

229. TE-306CL, sheath thermocouple, rod 6, level L .......... 140

230. TE-307CL, sheath therwocouple, $\operatorname{rod} 7$, level L ......... 140

231. TE-308CL, sheath therwocouple, $\operatorname{rod} 8$, level L .......... 141

232. TE-309EL, sheath therwocouple, rod 9, level L ........... 141

233. TE-310EL, sheath thernocouple, rod 10, level L ......... 142

234. TE-311CL, sheath thernocouple, $\operatorname{rod} 11$, level L ......... 142

235. TE-312EL, sheath therwocouple, $\operatorname{rod} 12$, level L .......... 143

236. TE-313EL, sheath thernocouple, rod 13, level L ......... 143

237. TE-315CL, sheath therwocouple, $\operatorname{rod} 15$, level L .......... 144

238. TE-316CL, sheath therwocouple, rod 16, level L ......... 144

239. TE-317EL, sheath therwocouple, $\operatorname{rod} 17$, level $1 \ldots \ldots \ldots \ldots 145$

240. TE-318EL, sheath thernocouple, $\operatorname{rod} 18$, level L ......... 145

241. TE-320EL, sheath thernocouple, rod 20, level $1 \ldots \ldots \ldots \ldots 146$

242. TE-321CL, sheath therwocouple, $\operatorname{rod} 21$, level L .......... 146

243. TE-322EL, sheath therwocouple, $\operatorname{rod} 22$, level $1 \ldots \ldots \ldots \ldots 147$

244. TE-323EL, sheath therwocouple, $\operatorname{rod} 23$, level $1 \ldots \ldots \ldots \ldots 147$

245. TE-324EL, sheath therwocouple, $\operatorname{rod} 24$, level $1 \ldots \ldots \ldots \ldots 148$

246. TE-325EL, sheath therwocouple, rod 25, level $1 \ldots \ldots \ldots \ldots . .148$

247. TE-326EL, sheath thermocouple, $\operatorname{rod} 26$, level L ......... 149

248. TE-327iL, sheath therwocouple, rod 27, livel L .......... 149

249. TE-323CL, sheath therwocouple, $\operatorname{rod} 28$, level L ......... 150

250. TE-331EL, sheath thermocouple, rod 31, level L .......... 150

251. TE-333EL, sheath therwocouple, $\operatorname{rod} 33$, level $L \ldots \ldots \ldots \ldots \ldots 151$

252. TE-336CL, sheath thermocouple, rod 36, level L .......... 151

253. TE-337CL, sheath thermocouple, $\operatorname{rod} 37$, level $L \ldots \ldots \ldots \ldots 152$ 
254. TE-338EL, sheath thermocouple, rod 38, level L ......... 152

255. TE-339EL, sheath thermocouple, rod 39, level L ......... 153

256. TE-340CL, sheati thernocouple, rod 40, level L .......... 153

257. TE-341EL, sheath thermocouple, rod 41, level L .......... 154

258. TE-342CL, sheath thermocouple, rod 42 , level L . . . . . . . . 154

259. TE-343CL, sheath thernocouple, rod 43, level L .......... 155

260. TE-344CL, sheath thermocouple, rod 44 , level L .......... 155

261. TE-345CL, sheath thermocouple, rod 45, livel L . . . . . . . 156

262. TE-346CL, sheath thernocouple, $\operatorname{rod} 46$, level L ......... 156

263. TE-347CL, sheath therwocouple, rod 47, level L ......... 157

264. TE-348CL, sheath thernozouple, rad 48, level L ......... 157

265. TE-349EL, sheath therrucouple, rod 49, level L ......... 158

266. TE-301EA, sheath thernocouple, rod 1, level M .......... 158

267. TE-304EH, sheath thermocouple, rod 4, level M ........... 159

268. TE-309EM, sheath thernocouple, rod 9, level M.......... 159

269. TE-325EH, sheath thermocouple, $\operatorname{rod} 25$, level $M \ldots \ldots \ldots \ldots \ldots$

270. TE-301FN, sheath thermocouple, rod 1, level $N \ldots \ldots \ldots \ldots \ldots 160$

271. TE-304FN, sheath thersocouple, $\operatorname{rod} 4$, level $N \ldots \ldots \ldots \ldots \ldots 1$

272. TE-325FN, sheath therwocouple, $\operatorname{rod} 25$, level $N \ldots \ldots \ldots \ldots 161$

273. TE-301F0, sheath thernocouple, rod 1, level $0 \ldots \ldots \ldots \ldots \ldots 16$

274. TE-304F0, sheath therwocouple, rod 4, level $0 \ldots \ldots \ldots \ldots \ldots 2$

275. TE-309F0, sheath thermocouple, $\operatorname{rod} 9$, level $0 \ldots \ldots \ldots \ldots 163$

276. TE-310E0, sheath thermocouple, $\operatorname{rod} 10$, level $0 \ldots \ldots \ldots \ldots 3$

277. TE-312EO, sheath thermocouple, rod 12, level $0 \ldots \ldots \ldots \ldots 164$

278. TE-317E0, sheath thermocouple, $\operatorname{rod} 17$, level $0 \ldots \ldots \ldots \ldots 164$

279. TE-318E0, sheath thermocouple, $\operatorname{rod} 18$, leve1 $0 \ldots \ldots \ldots \ldots$.....

280. TE-320E0, sheath thermoccuple, rod 20, level $0 \ldots \ldots \ldots \ldots . . .65$

281. TE-322E0, sheath thermocouple, $\operatorname{rod} 22$, level $0 \ldots \ldots \ldots \ldots 166$

282. TE-323E0, sheath thermocouple, rod 23. ievel $0 \ldots \ldots \ldots \ldots . . .166$

283. TE-324E0, sheath thermocouple, $\operatorname{rod} 24$, level $0 \ldots \ldots \ldots \ldots 167$

284. TE-325F0, sheath thermocouple, rod 25, level $0 \ldots \ldots \ldots \ldots 167$

285. TE-326E0, sheath thermocouple, $\operatorname{rod} 26$, level $0 \ldots \ldots \ldots \ldots 168$

286. TE-331E0, sheath thermocouple, rod 31, level $0 \ldots \ldots \ldots \ldots 168$

287. TE-333E0, sheath thermocolple, rod 33, level $0 \ldots \ldots \ldots \ldots 169$ 
288. TE-33850, sheath thereocouple, rod 38, level $0 \ldots \ldots \ldots \ldots . .169$

289. TE-339E0, sheach thernocouple, rod 39, level $0 \ldots \ldots \ldots \ldots .170$

290. TE-341E0, sheach thernocouple, rod 41, level $0 \ldots \ldots \ldots \ldots \ldots 170$

291. TE-349E0, sheath thermocouple, rod 49, level $0 \ldots \ldots \ldots \ldots \ldots 171$

292. TE-301ME, widdle therwocouple, rod 1, level E ........... 171

293. TE-318\%E, middle thermocouple, rod 18, level E ......... 172

294. TE-322KE, middle thernocouple, rod 22, level E ......... 172

295. TE-301MF, middle thernocouple, rod 1, level F .......... 173

296. TE-322MF, middle thermocouple, rod 22, !evel F ......... 173

297. TE-301MG, middle thermocouple, rod 1, level $G \ldots \ldots \ldots \ldots \ldots 174$

298. TE-3104G, middle thermocouple, rod 10, level G .......... 174

299. TE-317MG, middle thermocouple, rod 17, level G ......... 175

300. TE-318MG, middle thermocouple, rod 18, level G .......... 175

301. TE-322MG, middle thermocouple, $\operatorname{rod} 22,1$ evel G ......... 176

302. TE-323MG, middle thermpcouple, $\operatorname{rod} 23$, level $G \ldots \ldots \ldots \ldots \ldots 176$

303. TE-325MG, middle thermocouple, rod 25, level G .......... 177

-304. TE-304MH, middle thermocouple, rod 4, level H .......... 177

305. TE-369MH, middle thermocouple, rod 9, level H ........... 1;8

306. TE-318\%म, middle thermocouple, rod 18, level Н ......... 178

307. TE-322MH, middle therwocouple, $\operatorname{rod} 22$, level $\mathrm{H} \ldots \ldots \ldots \ldots \ldots 179$

308. TE-301M, middle thermocouple, rod 1, level J .......... 179

309. TE-303MJ, widdle therwocuuple, rod 3, level J .......... 180

310. TE-308MJ, middle thempocouple, rod 8, level J .......... 180

311. TE-310:J, middle therwocouple, rod 10, level J .......... 181

312. TE-317K, middle therwocouple, rod 17 , level $\mathrm{J} \ldots \ldots \ldots \ldots \ldots 1$

313. TE-322HL, middle thermocouple, rod 22, level J ......... 182

314. TE-323MJ, middie therwocouple, $\operatorname{rod} 23$, level $\mathrm{J} \ldots \ldots \ldots \ldots \ldots 182$

315. TE-324KJ, midale thermocouple, rod 24, level $\mathrm{J} \ldots \ldots \ldots \ldots \ldots 183$

316. TE-24, thermocouple, horizontal inlet spool piece ......... 183

317. TE-172, thermocruple, vertical inlet spool plece ......... 184

318. TE-222, thermoccuple, vertical outlet spool piece ......... 184

319. TE-40, thermocouple, horizontal outlet spool plece ........ 185

320. TE-162, test section Inlet line thermocouple ............ 185

321. TE-150, test section bottom flange thermocouple .......... 186 
322. TE-151, test section buttom flange thermocouple .......... 186

323. TE-152, test section botton flange thermocouple $\ldots \ldots \ldots \ldots \ldots 187$

324. TE-153, test section botton flange thermocouple .......... 187

325. TE-175, bundle shroud thermocouple, .142L/LWX .......... 188

326. TE-176, bundle shroud thermocouple, .142L/LMx .......... 188

327. TE-177, bundle shroud thermocouple, .142L/LWX .......... 189

328. TE-178, bundle shroud thernocouple, .142L/LMAX .......... 189

329. TE-179, bundle shroud therwocouple, .388L/LWAX .......... 190

330. TE-180, bundle shroud thermocouple, .388L/LMAX ......... 190

331. TE-181, bundle shroud thernocouple, .388L/LMx .......... 191

332. TE-182, bundle shroud therwocouple, .388L/LMAX .......... 191

333. TE-183, bundle shroud thernocouple, .633L/LMAX .......... 192

334. TE-184, bundle shroud therwocouple, .633L/LMAX .......... 192

335. TE-185, bundle shroud thermocouple, .633L/LYAX .......... 193

336. TE-186, bundle shroud thermocouple, .633L/LMAX .......... 193

337. TE-187, bundle shroud therzocouple, .875L/LAAX ........... 194

338. TE-189, bundle shroud thermocouple, .875L/LMAX .......... 194

339. TE-190, bundle shroud thermocouple, .875L/LAAX ........... 195

340. TE--12, test section outlet line thermocotiple ............ 195

341. TE-30B, heat exchanger A out let thermocouple ........... 196

342. TE-32B, heat exchanger B out let thermocouple ............ 196

343. TE-34B, heat exchanger $C$ out let thermocouple ........... 197

344. TE-520B, RBT 4 in. demineralized water header thermocouple ....................................... 197

345. TE-5B, heat exchanger D outlet thermocouple $\ldots \ldots \ldots \ldots \ldots \ldots 198$

346. TE-1, pressurizer tank top thermocouple .............. 198

347. TE-2, pressurizer tank exit thermocouple ............... 199

348. TE-29, inlet blowdown plenum thermocouple .............. 199

349. TE-45, outlet blowdown plenum thermocouple ............ 200

350. TE-4B, base primary steady-state temperature, RTD ........ 200

351. FE-620, HX B secondary cooling water flow ............ 201

352. FE-720, HX C secondary cooling water flow ............. 201

353. FE-550, HX D secondary cooling water flow ............. 202

354. FE-1A, primary side pump flow ................... 202 
355. FE-19, turbine flow meter, horizontal inlet spool piece .... 203

356. FE-166, turbine flow meter, vertical inlet spool piece ..... 203

357. FE-216, turbine flow neter, vertical outlet spool piece .... 204

358. FE-34, turbine flow meter, horizontal outlet apool piece ... 204

359. PE-26, transient pressure, horizontal inlet spool piece .... 205

360. PE-174, transient pressure, vertical inlet spool plece .... 205

361. PE-156, test section inlet plenum transient pressure ...... 206

362. PE-201, test section outlet plenu= transient pressure ..... 206

363. PE-224, transient pressure, vertical outlet spool piece .... 207

364. PE-42, transient pressure, horizontal outlet spool piece ... 207

365. PE-44, upstrean main HX transient pressure ............. 208

366. PE-76, primary punp suction transtent pressure .......... 208

367. PT-102, pressurizer steady-state pressure .............. 209

368. PE-106, pressurizer vapor transient pressure ............ 209

369. PE-412, pressure suppression recetver transient pressure ... 210

370. PDE-167, transient DP, vertical inlet spool piece ........ 210

371. TE-615, demineralized water $6 \mathrm{in}$. header steady-state teap. ...................................... 211

372. PDT-199, test section shroud box steady-state DP ........ 211

373. PDE-200, test bundle transient DP ................ 212

374. PDE-35, transient DP, horizontal outlet spool piece ....... 212

375. PDE-217, transienc DP, vertical outlet spool piece ........ 213

376. TE-627, HX B secondary discharge steady-state temp., RTD ... 213

377. PDT-48, main HX steady-state DP ................. 214

378. PDE-78, primary pump transient DP ................ 214

379. PE-425, PSS inlet blowdown line transient pressure ....... 215

380. PE-427, PSS outlet blowdown line transient pressure ...... 215

381. TE-727, HX C secondary discharge steady-state temp., RTO ... 216

382. TE-28B, main HX mixing tee steady-state temp., RTD ....... 216

383. PDE-46, mafn HX bypass transient DP $\ldots \ldots \ldots \ldots \ldots \ldots \ldots \ldots \ldots 217$

384. FMPE-22, drag disk, horizontal inlet spool piece ......... 217

385. FMFE-170, drag disk, vertical inlet spool plece ......... 218

386. FMFE-220, drag disk, vertical outlet spool plece ......... 218

387. FMPE-38, drag disk, horizontal outlet spool piece ........ 219 
388. PE-525, HX A secondary inlet pressure ............... 219

389. PDE-21, transient DP, horizontal inlet spool piece ........ 220

390. PE-16, downstrean HCV-2 transient pressure ............. 220

391. PE-27, test section inlet plenum botton transient pressure ...................................... 221

392. PE-43, test section outlet plenum botton transfent pressure ....................................... 221

393. PDT-30, test section steady-state DP $\ldots \ldots \ldots \ldots \ldots \ldots \ldots \ldots 22$

394. PT-32, test section outlet steady-state pressure ......... 222

395. SE-72, prinary punp speed ...................... 223

396. XY-40001, breakwire detector, inlet break ............. 223

397. Xr-4000, breakwire detector, outlet break ............ 224

398. LT-100, steady state pressurizer liquid level ........... 224

399. FE-522, HX A secondary cooling water flow .............. 225

400. TE-557, HX D secondary discharge steady-state temp., RTD ... 225

401. TE-525, HX A secondary discharge steady-state temp., RTD ... 226

402. TE-210A, test section outlet line steady-state temp., RTD .. 226

403. EIM-1001B, RTD power supply current ................. 227

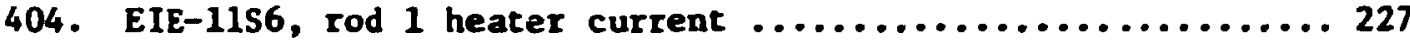

405. EIE-12S6, rod 2 heater current .................. 228

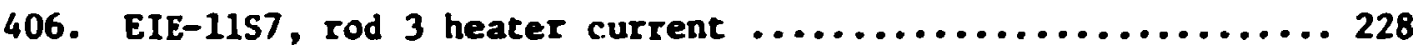

407. EIE-12S7, rod 4 heater current ................... 229

408. EIE-9S6, rod 5 heater current .................... 229

409. EIE-9S8, rod 6 heater current $\ldots \ldots \ldots \ldots \ldots \ldots \ldots \ldots \ldots \ldots \ldots$

410. EIE-9S5, rod 7 heater current $\ldots \ldots \ldots \ldots \ldots \ldots \ldots \ldots \ldots \ldots \ldots$

411. EIE-1155, rod 8 heater current $\ldots \ldots \ldots \ldots \ldots \ldots \ldots \ldots \ldots \ldots 231$

412. EIE-11S8, rod 9 heater current $\ldots \ldots \ldots \ldots \ldots \ldots \ldots \ldots \ldots \ldots \ldots \ldots$

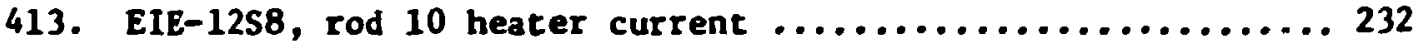

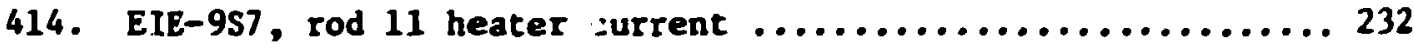

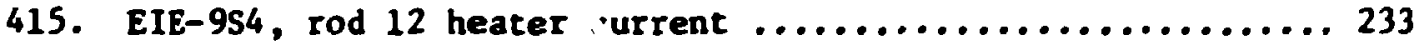

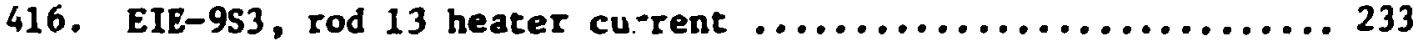

417. ElE-9S11, rod 14 heater current .................. 234

418. EIE-11S4, rod 15 heater current .................. 234

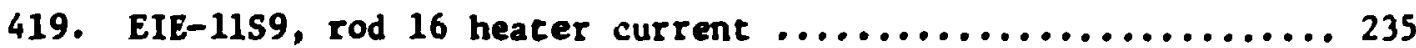


xvi

Page

420. EIE-12S5, $\operatorname{rod} 17$ heater current $\ldots \ldots \ldots \ldots \ldots \ldots \ldots \ldots \ldots . \ldots \ldots$

421. EIE-9S9, rod 18 heacer current $\ldots \ldots \ldots \ldots \ldots \ldots \ldots \ldots \ldots \ldots \ldots .236$

422. EIE-9S10, rod 19 heater current .................. 236

423. EIE-9S2, rod 20 heater current $\ldots \ldots \ldots \ldots \ldots \ldots \ldots \ldots \ldots . \ldots \ldots$

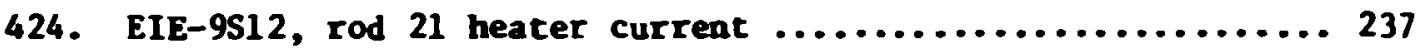

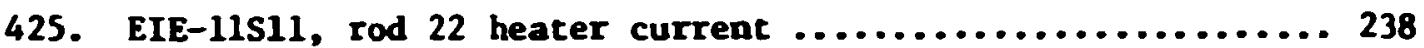

426. EIE-11510, rod 23 heater current $\ldots \ldots \ldots \ldots \ldots \ldots \ldots \ldots \ldots . \ldots \ldots$

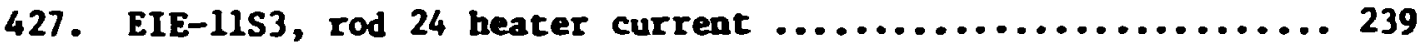

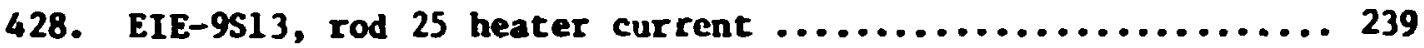

429. EIE-10S6, $\operatorname{rod} 26$ heater current $\ldots \ldots \ldots \ldots \ldots \ldots \ldots \ldots \ldots . \ldots . \ldots . \ldots$

430. EIE-9S1, $\operatorname{rod} 27$ heater current $\ldots \ldots \ldots \ldots \ldots \ldots \ldots \ldots \ldots . \ldots . \ldots \ldots$

431. EIE-10S7, rod 28 heater current $\ldots \ldots \ldots \ldots \ldots \ldots \ldots \ldots \ldots . \ldots \ldots 1$

432. EIE-11S2, $\operatorname{rod} 29$ heater current $\ldots \ldots \ldots \ldots \ldots \ldots \ldots \ldots \ldots . \ldots \ldots 1$

433. EIE-11S12, rod 30 heater current $\ldots \ldots \ldots \ldots \ldots \ldots \ldots \ldots . \ldots 242$

434. EIE-12S2, rod 31 heater current $\ldots \ldots \ldots \ldots \ldots \ldots \ldots \ldots \ldots . \ldots 242$

435. EIE-12S1, rod 32 heater current $\ldots \ldots \ldots \ldots \ldots \ldots \ldots \ldots \ldots . \ldots \ldots$

436. EIE-10S12, rod 33 heater current $\ldots \ldots \ldots \ldots \ldots \ldots \ldots \ldots \ldots . \ldots . \ldots . \ldots$

437. EIE-10S5, rod 34 heater current ................... 244

438. EIE-10S8, rod 35 heater current $\ldots \ldots \ldots \ldots \ldots \ldots \ldots \ldots \ldots . \ldots . \ldots \ldots$

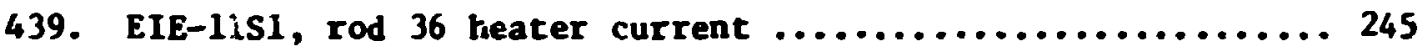

440. EIE-12S4, rod 37 heater current $\ldots \ldots \ldots \ldots \ldots \ldots \ldots \ldots \ldots \ldots . \ldots \ldots$

441. EIE-12S3, rod 38 heater current .................. 246

442. EIE-12S12, rod 39 heater current $\ldots \ldots \ldots \ldots \ldots \ldots \ldots \ldots \ldots$

443. EIE-10S11, rod 40 heater current ................. 247

444. EIE-10S10, rod 41 heater current $\ldots \ldots \ldots \ldots \ldots \ldots \ldots \ldots . \ldots 247$

445. EIE-10S9, rod 42 heater current ................... 248

446. EIE-12S9, rod 43 heater current ................... 248

447. EIE-12S10, rod 44 heater current ................. 249

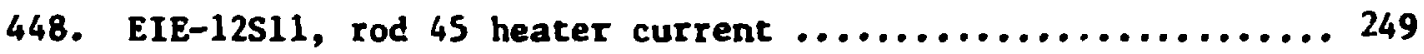

449. EIE-10S1, rod 46 heater current $\ldots \ldots \ldots \ldots \ldots \ldots \ldots \ldots \ldots . \ldots 250$

450. EIE-10S2, rod 47 heater current $\ldots \ldots \ldots \ldots \ldots \ldots \ldots \ldots \ldots \ldots . \ldots 250$

451. EIE-10S3, rod 48 heater current $\ldots \ldots \ldots \ldots \ldots \ldots \ldots \ldots \ldots \ldots$

452. EIE-10S4, rod 49 heater current ................... 251 


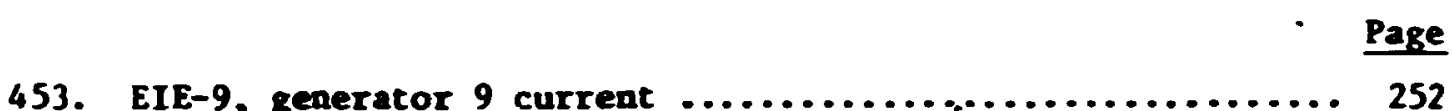

454. EIE-10, generacor 10 current ...................... 252

455. EIE-11, geuerator 11 current ...................... 253

456. EIE-12, generator 12 current .................... 253

457. EEE-9, gererator 9 voltage ....................... 254

458. EEE-10, generator 10 voltage $\ldots \ldots \ldots \ldots \ldots \ldots \ldots \ldots \ldots \ldots . \ldots . \ldots . \ldots$

459. EEE-11, generator 11 voltage ..................... 255

460. EEE-12, generator 12 voltage ...................... 255

461. DE-20, gama densitoneter, horizontal inlet spool piece .... 256

462. DE-168, zam densitometer, vertical inlet apool piece .... 256

463. DE-218, gana densitometer, vertical outlet spool piece .... 257

464. DE-36, gama densitometer, horizontal outlet spool plece ... 257

465. Zero calibration input, channels $0-127 \ldots \ldots \ldots \ldots \ldots \ldots \ldots . . \ldots 258$

466. Zero calibration input, channels 128-255 ............... 258

467. Zero calib:ation input, channels $256-383 \ldots \ldots \ldots \ldots \ldots \ldots . . .259$

468. Zero calibiation input, channels $384-511 \ldots \ldots \ldots \ldots \ldots \ldots . . \ldots 259$

469. Full-scale calibration input, channels $0-127 \ldots \ldots \ldots \ldots \ldots \ldots$

470. Full-scale calibration input, channels $128-255 \ldots \ldots \ldots \ldots . .260$

471. Full-scale calibration input, channels 256-383 ......... 261

472. Ful1-scale calibration input, channels $384-511 \ldots \ldots \ldots \ldots . . .261$

473. TE-S1, subchannel 1 thermocouple .................. 262

474. TE-S2, subchannel 2 thermocouple ................. 262

475. TE-S3, subchannel 3 therwocouple .................. 263

476. TE-\$4, subchannel 4 therwocouple .................. 263

477. 1E-S5, subchannel 5 thermocouple .................. 264

478. TE-S6, subchanncl 6 thermocouple .................. 264

419. TE-S7, subchannel 7 therwocouple .................. 265

480. TE-S8, subchannel 8 the:mocouple ................... 265

481. TE-S9, subchannel 9 thermocouple ................... 266

482. TE-S10, subchannel 10 therwocouple .................. 266

483. TE-S11, subchannel 11 therwocouple ................... 267

484. TE-S13, subchannel 13 thermocouple ................. 267

485. TE-S17, subchannel 17 thermocouple ................. 268

486. TE-S18, subchannel 18 thermocouple ................. 268 
487. TE-S19, subchnnel 19 thernocouple ................... 259

488. TE-S20, subchannel 20 thermocouple ................... 269

489. TE-S25, subchannel 25 thermocouple .................. 270

490. TE-S26, subchanael 26 therwocouple ................... 270

491. TE-S27, subchannel 27 thermocouple .................. 27]

492. TE-S28, subchannel 28 thermocouple .................. 271

493. TE-S29, subchnnel 29 therwocouple $\ldots \ldots \ldots \ldots \ldots \ldots \ldots \ldots \ldots . \ldots 272$

494. TE-S30, subchannel 30 therwocouple ................... 272

495. TE-s31, subchandel 31 thermocnuple ................. 273

496. TE-S32, subchannel 32 thernocouple $\ldots \ldots \ldots \ldots \ldots \ldots \ldots \ldots \ldots . \ldots . \ldots . \ldots$

497. TE-S33, subchannel 33 thernocouple .................. 274

498. TE-S34, subchannel 34 thernocouple ................. 274

499. TE-S35, subchannel 35 thernocouple .................. 275

500. TE-S36, subchannel 36 thermocouple ................ 275

501. TE-S37, subchannel 37 thernocouple ................ 276

502. TE-S46, subchannel 46 thernocouple ................... 276

503. TE-S55, subchannel 55 thernocouple ................ 277

504. TE-S64, subchannel 64 thernocouple ................. 277 


\section{ACKMOULEDGIENTS}

In the conduct of a large experimental and analytical progran, there are always a great any individuals whose contributions should be recognized. The dedicated efforts of the entire BDHT progran staff are reflected in this report. The authors express their appreciation to the following personnel:
H. C. Adair
R. H. Greene
J. L. Redford
W. E. Baucum
R. C. Hagar
M. J. Roberts
R. E. Bohanan
R. E. Helss
L. I. Schlemper
S. B. Cliff
C. R. Hyman
A. N. Saith
C. E. Davis
P. A. Jallouk
R. D. Stulting
E. D. Drennen
A. F. Johnson
R. E. Textor
B. G. Eads
D. M. Leon
D. G. Thomas
G. G. Fee
G. S. Masseng 111
M. L. Walker
D. J. Fraysier
L. J. Ott
J. D. White
R. M. Fl anders
H. R. Payne
J. E. Wolfe
C. A. Gifford
H. Ragan, Jr. 
PRR BLOWDONA HEAT TRANSFER SEPARATE-EFTECTS

PROGRAM - THERAAL-ETDRAULIC TEST FACILIT

EXPERIRLATAL DATA REPORT FOR TEST 103

\author{
V. D. Clemons \\ P. A. Hoore \\ M. D. White \\ R. A. Hedrick
}

\begin{abstract}
Reduced instrunent responses are presented for ThernalHydraulic Test Facility (THTF) test 103, which is part of the ORNL Pressurized-later Reactor (PWR) Bloudown Heat Transfer Separate-Ef fects Program. The objective of the progran is to investigate the theral-hydraulic phenomenon goversing the energy transfer and transport procesees that occur during a loss-of-coolant accident in a PWR systen.

Test 103 was conducted to obtain design information for THTP test 105.

The prinary purpose of this report is to ake the reduced instrument responses during test 103 available. These are presented in grishical form in engineering units and have been analyzed only to the extent necessary to ensure reasonableness and consistency.
\end{abstract}

\title{
I. INTRODUCTION
}

The Oak Ridge National Laboratory Pressurized-Water Reactor (ORNL-PWR) Blowdown Heat Transfer Program is a separate-effects study of the relations among the principal variables that can alter the rate of blowdown, the presence of flow reversal and rereversal, time delay to critical heat flux (CHF), the rate at which dryout progresses, and imilar time- and spacerelated functions that are important in 10ss-of-conlant accident (LOCA) analyses. Primary test results are obtained from the Thermal-Hydraulic Test Facilfty (THTF), a large nonnuclear pressurized-water loop incorporating a 49-rod electrically heated bundle in $7 \times 7$ geometry.

THTF test 103 (conducted August 5, 1976) was the fifth test conducted in the facility with bundle 1 in place. This test was performed to provide experimental verification of the RELAP4 prediction of epool piece flows with a 407 inlet -607 outlet break.

The purpose of this report is to provide the reduced instrument responses during test 103 in a readily usable form to the nuclear community 
In advance of detailed analyses and interpretations. (These data are preseated on aicrofiche attached to the back cover of the report.) Final analyses and interpretations are scheduled for publication afx wonth after the conpletion of teat series 100. The progra and the experinental facilIties are described in Ref. 1 .

\section{STSTIS, PROCEDURES, CONDITIOAS, ND EVEITS FOR TEST 103}

\section{Syste Confiruration and Test Procedure}

The Theral-Hydraulic Test Facility (TtIF), shown in Fig. 1, consists of a test section with a 49-rod, 3.66-0-10ng (12-ft) electrically bented core; a circulation loop coprised of three parallel beat exchangers with bypass, a pressurizer, a pup with bypass, and asecciated control valves: two rupture assebblies; and a pressure-suppression aysten. The break configuration for teat $103 \mathrm{mas} 40 \mathrm{f}$ inlet-607 outlet with a total break area of $12.54 \mathrm{~cm}^{2}\left(0.0135 \mathrm{ft}^{2}\right)$. The Tirr experimental syaten is described further in Ref. 1.

The electric core was taken to the preblowdown power (122 kH/rod) in 220-ky/rod steps to provide steady-state calibration information. The ain heat exchangers were operated to watch the core power input. The prinary coolant purp was tripped coincident with break initiation, but the electic core was operated at the preblowdown power for $\eta_{2}$ sec into the transient. Closure of the secondary-side matn beat exchanger valves was initlated at the trip from $122 \mathrm{kH} / \mathrm{rod}$.

In preparation for the teat, the loop was filled with denfnerallzed water and the systen pressure checked. Instrumentation and data acquisitlon checke were perforned. During the warmup, data were taken for use In flow and preseure calibrations.

During the test, the THIP was successfully subjected to a double-ended pipe break through the rupture asseablies containing the orifice plates.

1. Project Desoription: ORILPLR BLowdonn Heat Treorofer Separate-

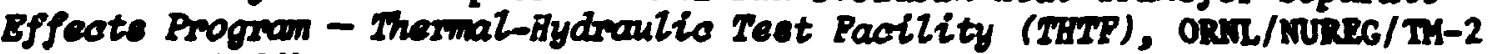
(February 1976). 


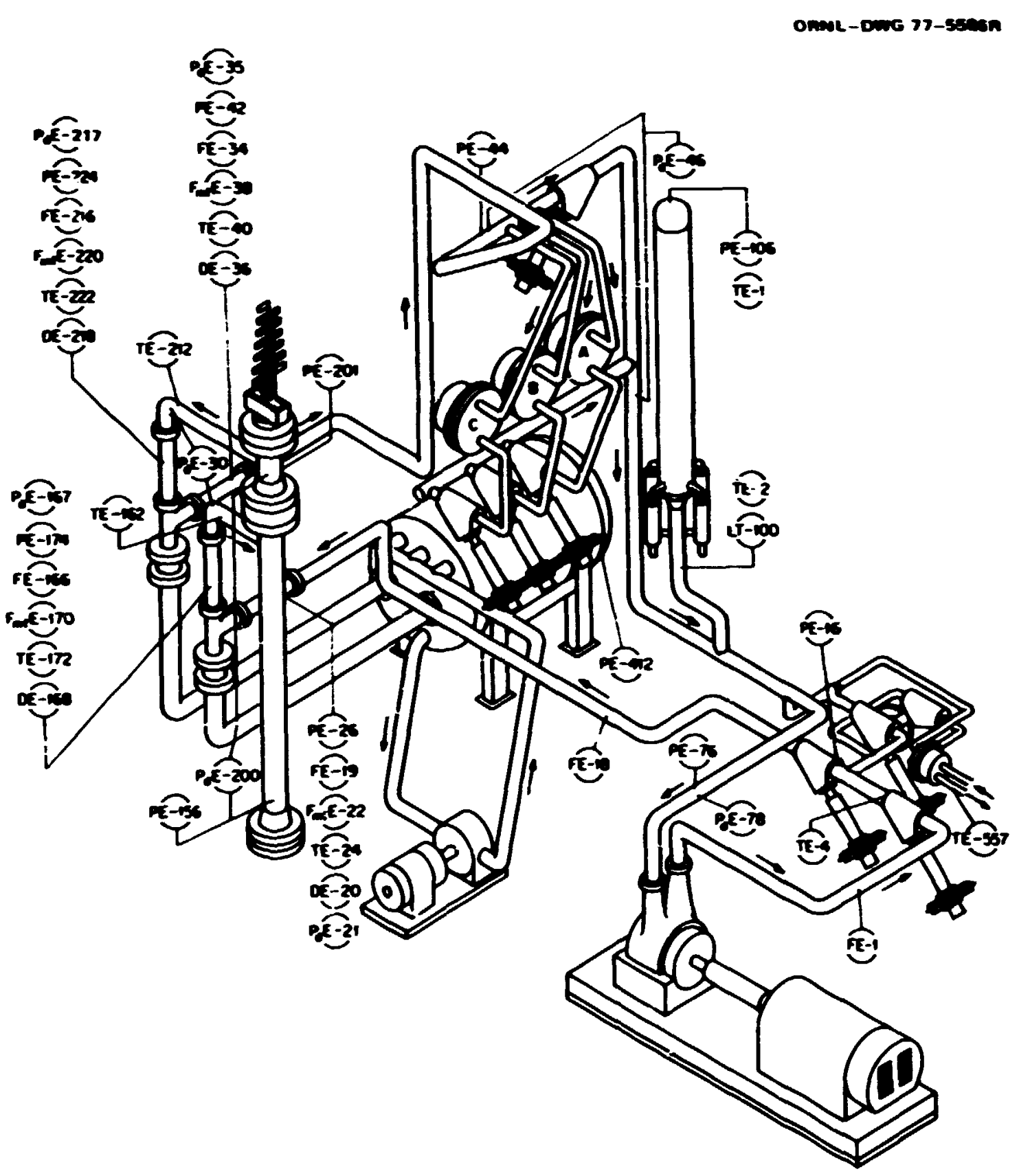

F18. 1. Thermal-Hydraulic Test Facility (ThTF). 
The effluent fron the prinary oysten was ejected into the pressuresuppression systen, which was mincained at atmospheric pressure. The priang-coolant pup was tripped colncident with break initiation.

\section{Initial Test Conditions and Sequence of Events}

The THIF conditions imediately preceding rupture are given in Tables 1 and 2. The sequence of events relative to the rupture is given in Table 3.

Table 1. Desited wo actual pretupture coadicions

\begin{tabular}{|c|c|c|c|}
\hline Parmeres & Instrumenc & Desired & Accuel ${ }^{b}$ \\
\hline $\begin{array}{l}\text { syecen pressure } \\
m / a^{2} \\
\mathrm{mois}\end{array}$ & $P E-201$ & $\begin{array}{l}15.513 \\
2250\end{array}$ & $\begin{array}{l}15.561 \\
2257\end{array}$ \\
\hline $\begin{array}{l}\text { Core power } \\
\text { ind }\end{array}$ & $\begin{array}{l}\text { EIE-9, EIE-10, EIE-11, EIE-12 } \\
\text { EEE-9, EEE-10, EEE-11, EEE-12 }\end{array}$ & 5.978 & 5.975 \\
\hline $\begin{array}{l}\text { Core volumetric flow rate } \\
\text { isec } \\
\text { epe }\end{array}$ & FE-19 & $\begin{array}{l}0.0265 \\
620\end{array}$ & $\begin{array}{l}0.027 \\
627\end{array}$ \\
\hline $\begin{array}{l}\text { Test section inlet tempertacure } \\
\text { ! } \\
\bullet_{F}\end{array}$ & $\pi E-162$ & $\begin{array}{l}559.3 \\
567\end{array}$ & $\begin{array}{l}550.2 \\
545\end{array}$ \\
\hline $\begin{array}{l}\text { Test section out let temperature } \\
\text { I } \\
{ }_{F} \mathrm{~F}\end{array}$ & TE-212 & $\begin{array}{l}610.4 \\
639\end{array}$ & $\begin{array}{l}606.5 \\
632\end{array}$ \\
\hline $\begin{array}{l}\text { Prescurizer preseure } \\
\text { mila } \\
\text { pois }\end{array}$ & $P E-106$ & $\begin{array}{l}15.031 \\
2180\end{array}$ & $\begin{array}{l}15.306 \\
2220\end{array}$ \\
\hline $\begin{array}{l}\text { Mase liquid wacer } \\
\text { kg } \\
\text { lb }\end{array}$ & & $\begin{array}{l}63.50 \\
160\end{array}$ & $\begin{array}{l}58.94 \\
129.94\end{array}$ \\
\hline $\begin{array}{l}\text { Coolant pup speed } \\
\text { rps } \\
\text { rpan }\end{array}$ & $S E-72$ & $\begin{array}{l}59.67 \\
3580\end{array}$ & $\begin{array}{l}60.12 \\
3607\end{array}$ \\
\hline $\begin{array}{l}\text { Preasure differencial } \\
\mathrm{m} / \mathrm{m}^{2} \\
\text { pold }\end{array}$ & $P_{d} E-78$ & $\begin{array}{l}4.613 \\
660\end{array}$ & $\begin{array}{l}4.475 \\
649\end{array}$ \\
\hline $\begin{array}{l}\text { Preseure between } \mathrm{HCV}-2 \text { and } \mathrm{FCV}-18 \\
\mathrm{pu/m} / \mathrm{m}^{2} \\
\mathrm{psis}\end{array}$ & $P E-16$ & $\begin{array}{l}16.996 \\
2665\end{array}$ & $\begin{array}{l}16.927 \\
2455\end{array}$ \\
\hline $\begin{array}{l}\text { Preseure differencial ocross anin } \\
\text { heat exchengers } \\
\text { mo/s } \\
\text { poid }\end{array}$ & $P_{d} E-66$ & $\begin{array}{l}0.172 \\
25\end{array}$ & $\begin{array}{l}0.165 \\
24\end{array}$ \\
\hline
\end{tabular}


Table 2. Prerupture primary-coglant ceperature and pressure distribution test 103

\begin{tabular}{|c|c|c|c|c|}
\hline Location & Instrument & $\begin{array}{l}\text { Texpereture } \\
\left|\mathrm{R}\left({ }^{\circ}\right)\right|\end{array}$ & $\begin{array}{l}\text { Pres } \\
\text { itmote }\end{array}$ & (posis)] \\
\hline $\begin{array}{l}\text { Vertical inlet spool piece } \\
\text { Vertical inlet spool piece }\end{array}$ & $\begin{array}{l}\text { TE-172 } \\
\text { PE-176 }\end{array}$ & $558.7(546)$ & 15.796 & $(2291)$ \\
\hline Test section inlet & TE-162 & $558.2(545)$ & & \\
\hline $\begin{array}{l}\text { Lower plenu } \\
\text { lower plenus }\end{array}$ & $\begin{array}{l}\text { TE-150 } \\
\text { PE-156 }\end{array}$ & $560.9(550)$ & 15.755 & $(2285)$ \\
\hline Upper plenu & PE-201 & & 15.561 & $(2247)$ \\
\hline Test section outlet & TE-2:2 & $606.5(632)$ & & \\
\hline $\begin{array}{l}\text { Vertical outlet spool piece } \\
\text { Vertical outlet spool piece }\end{array}$ & $\begin{array}{l}\text { TE-222 } \\
\text { PE-224 }\end{array}$ & $606.5(632)$ & 15.458 & $(2242)$ \\
\hline Heat exchanger inlet header & $P E-46$ & & 15.396 & $(2233)$ \\
\hline $\begin{array}{l}\text { Mixed wean ceeperature } \\
\text { dounstrean beat exchangers }\end{array}$ & TE-28B & $564.8(557)$ & & \\
\hline $\begin{array}{l}\text { Pressurizer surge line } \\
\text { Pressurizer }\end{array}$ & $\begin{array}{l}\text { TE-2 } \\
\text { PE-106 }\end{array}$ & $544.8(521)$ & 15.306 & $(2220)$ \\
\hline Primary pup suction & $P E-76$ & & 15.279 & $(2216)$ \\
\hline $\begin{array}{l}\text { Detween main control valves } \\
\text { HCV-2, FCV-18 }\end{array}$ & TE-4B & $556.5(542)$ & & \\
\hline $\begin{array}{l}\text { Detween main control yalves } \\
\text { HCV-2, FCV-18 }\end{array}$ & PE-16 & & 16.927 & (2655) \\
\hline
\end{tabular}

Table 3. Sequence of events during cest 103

\begin{tabular}{lr}
\hline \multicolumn{1}{c}{ Event } & $\begin{array}{c}\text { Time relative } \\
\text { to rupture } \\
\text { (sec) }\end{array}$ \\
\hline Core power level established & -4920 \\
Core teaperature rise established & -4920 \\
Analog capes and CcDas fast scan started & -15 \\
Blowdown initiated & 0 \\
Pump power tripped & 0 \\
Heat exchanger secondary val ves closife initiated & +2 \\
Core power tripped & +2 \\
\hline
\end{tabular}




\section{DATA PRESEnTATIOA}

The recorded instrument responses for THTF test 103 have been processed only to the extent necessary to obtain appropriate engineering units and to ensure reasonableness and consistency. In converting the instrunent responses to engineering units, a hongeneous fluid has been assuned. Therefore, interpretation or analysis of the data must account for the fact that the instruments may have been subjected to nonhomogeneous fluid conditions during the transient.

The reduced instrument responses presented in this report were recorded by a computer-controlled digital data acquisition system (CCDAS) . Further information on this system ay be found in Ref. 1.

Figures 2 through 4 provide supportive information for the instrument responses and indicate the relative locations of the detectors in the THTF. Table 4 gives the precision of the recorded instrument responses, and Table 5 groups the measurements by location and provides brief coments regarding the detectors and the recorded resfonses. Time zero on all graphs is the time of break initiation.

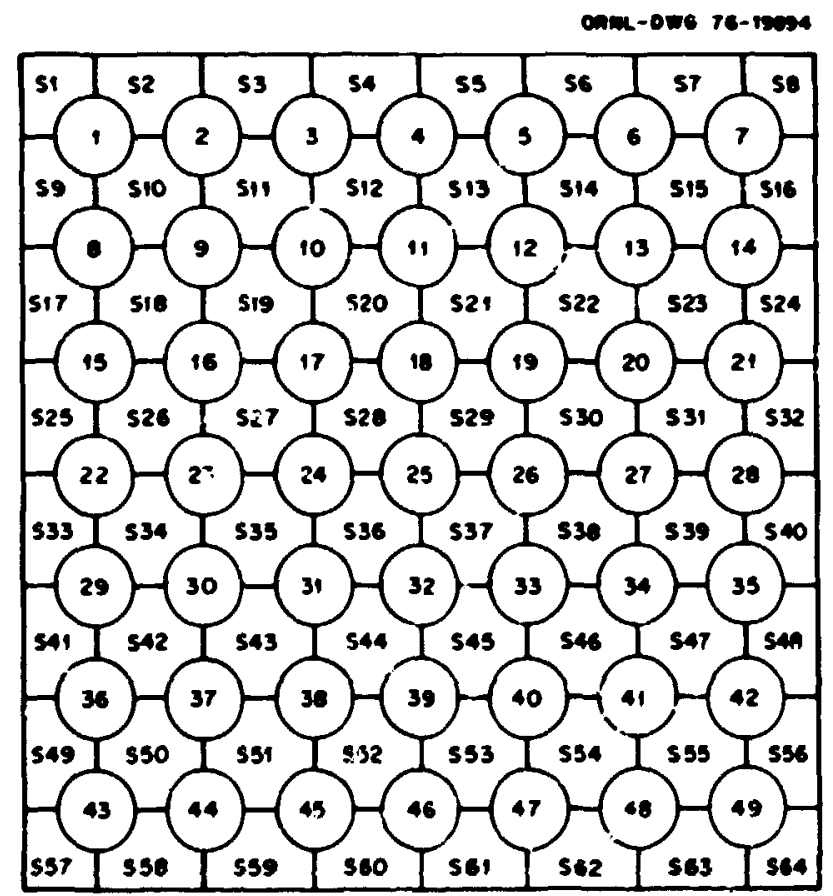

F1g. 2. Identification of THTE heater rod and subchannel locations In bundles 1 and 2 . 
Table 4. Precision of experimental measurements for test 103

\begin{tabular}{|c|c|}
\hline Systee & Standard teviation \\
\hline \multicolumn{2}{|l|}{ Pressure measurement, $\mathrm{kN} / \mathrm{m}^{2}$ (psig) } \\
\hline $\begin{array}{l}\text { ccous } \\
\text { Analog tape system }\end{array}$ & $\begin{array}{ll}0.185 & (26.8) \\
0.197 & (28.5)\end{array}$ \\
\hline \multicolumn{2}{|l|}{ Pressure difference weasurement, $\mathrm{ks} / \mathrm{m}^{2}$ (psid) } \\
\hline 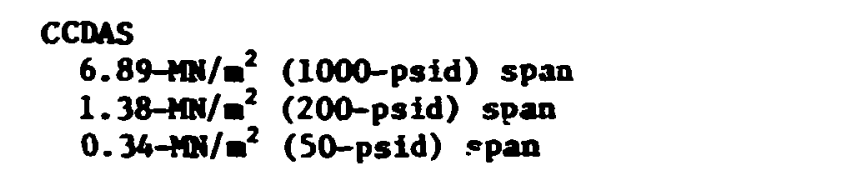 & $\begin{array}{ll}0.025(3.6) \\
0.005(0.72) \\
0.001(0.18)\end{array}$ \\
\hline 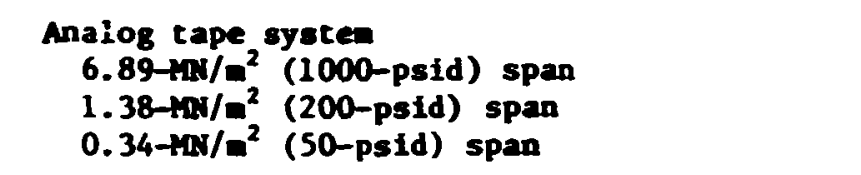 & $\begin{array}{l}0.033(4.8) \\
0.007(0.95) \\
0.002(0.24 !\end{array}$ \\
\hline Temperature measurement, $K\left({ }^{\bullet} F\right)$ & $2.4(4.3)$ \\
\hline \multicolumn{2}{|l|}{ Electric core power measurement } \\
\hline $\begin{array}{l}\text { Rod current, } \mathbf{A} \\
\text { Rod voltage, } \mathbf{v}\end{array}$ & $\begin{array}{l}0.877 \\
0.304\end{array}$ \\
\hline \multicolumn{2}{|l|}{ Flow measurewent, $\mathrm{m}^{3} / \mathrm{sec}$ (gpm) } \\
\hline $\begin{array}{l}\text { FE-19 } \\
\text { Forward } \\
\text { Reverse }\end{array}$ & $\begin{array}{l}+0.0009-0.0002 \\
(+13.97)(-2.90) \\
+0.0011-0.0004 \\
(+16.77)(-5.70)\end{array}$ \\
\hline \multicolumn{2}{|l|}{ FE-166 } \\
\hline Forward & $\begin{array}{l}+0.0012-0.0005 \\
(+18.74)(-7.68)\end{array}$ \\
\hline Reverse & $\begin{array}{l}+0.0010-0.0003 \\
(+16.15)(-5.09)\end{array}$ \\
\hline \multicolumn{2}{|l|}{ FE-216 } \\
\hline $\begin{array}{l}\text { Forward } \\
\text { Reverse }\end{array}$ & $\begin{array}{l}+0.0048-0.0041 \\
(+75.59)(-64.52) \\
+0.0020-0.0013 \\
(+31.79)(-20.72)\end{array}$ \\
\hline $\begin{array}{l}\text { FE-34 } \\
\text { Forward } \\
\text { Reverse }\end{array}$ & $\begin{array}{l}+0.0021-0.0007 \\
(+33.39)(-11.26) \\
+0.0124-0.0110 \\
(+197.11)(-174.97)\end{array}$ \\
\hline \multicolumn{2}{|l|}{$\begin{array}{l}\text { Mowentum flux measurement, } \mathrm{kg} / \mathrm{m}-\sec ^{2} \\
\left(1 \mathrm{~b}_{\mathrm{m}} / \mathrm{ft}-\sec ^{2}\right)\end{array}$} \\
\hline $\begin{array}{l}\text { CCDAS } \\
\text { Analo8 tape system }\end{array}$ & $\begin{array}{l}6793(4565) \\
7661(5148)\end{array}$ \\
\hline $\begin{array}{l}\text { Density measurement o } 961 \mathrm{~kg} / \mathrm{m}^{3}\left(601 \mathrm{~b}_{\mathrm{m}} / \mathrm{ft}^{3}\right) \text {, } \\
\mathrm{kg} / \mathrm{m}^{3}\left(1 \mathrm{~b}_{\mathrm{m}} / \mathrm{ft}^{3}\right)\end{array}$ & $12.9(0.81)$ \\
\hline
\end{tabular}




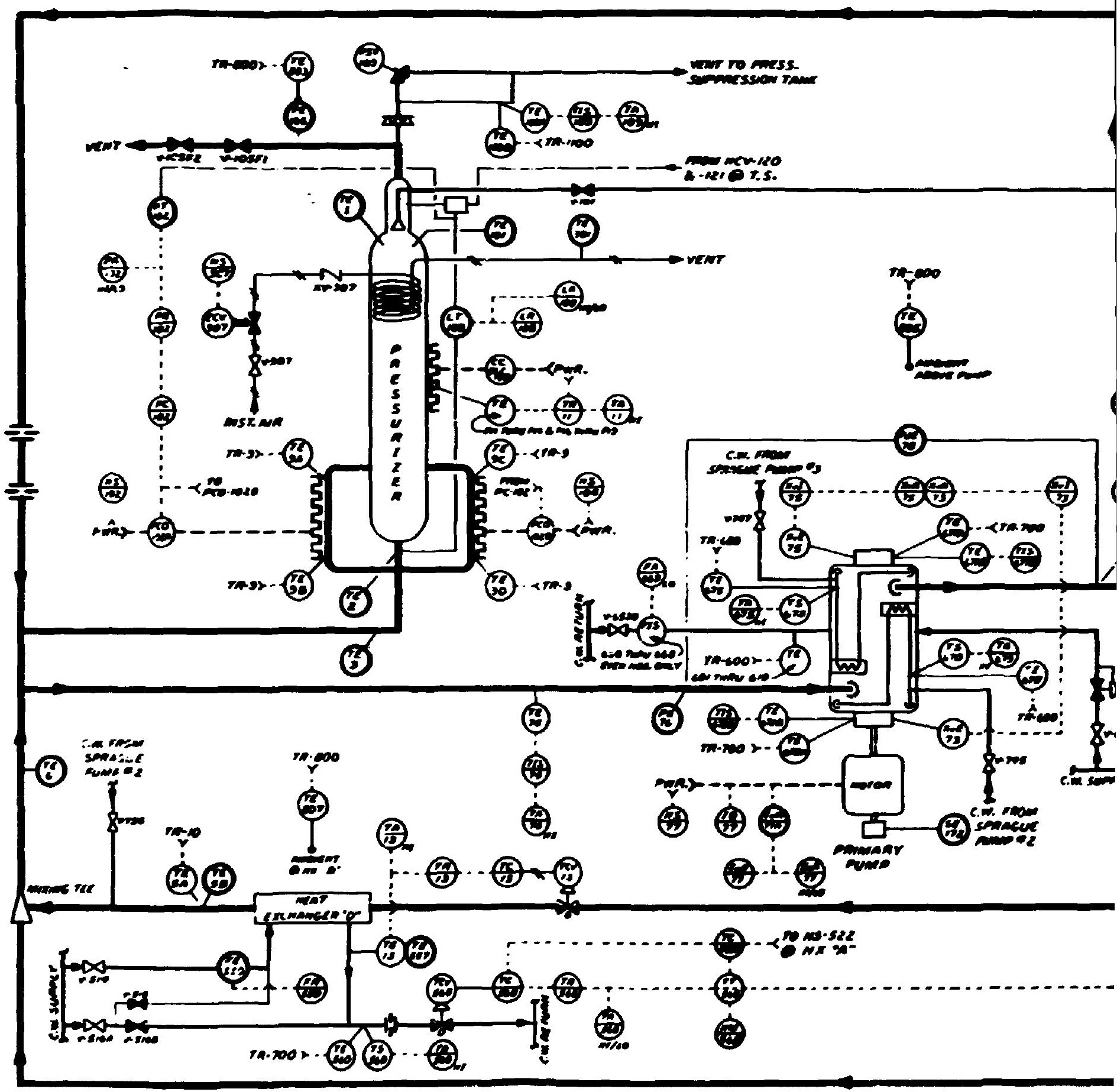

Fig. 3. Location of thermocouples in THTF bundle 1. 


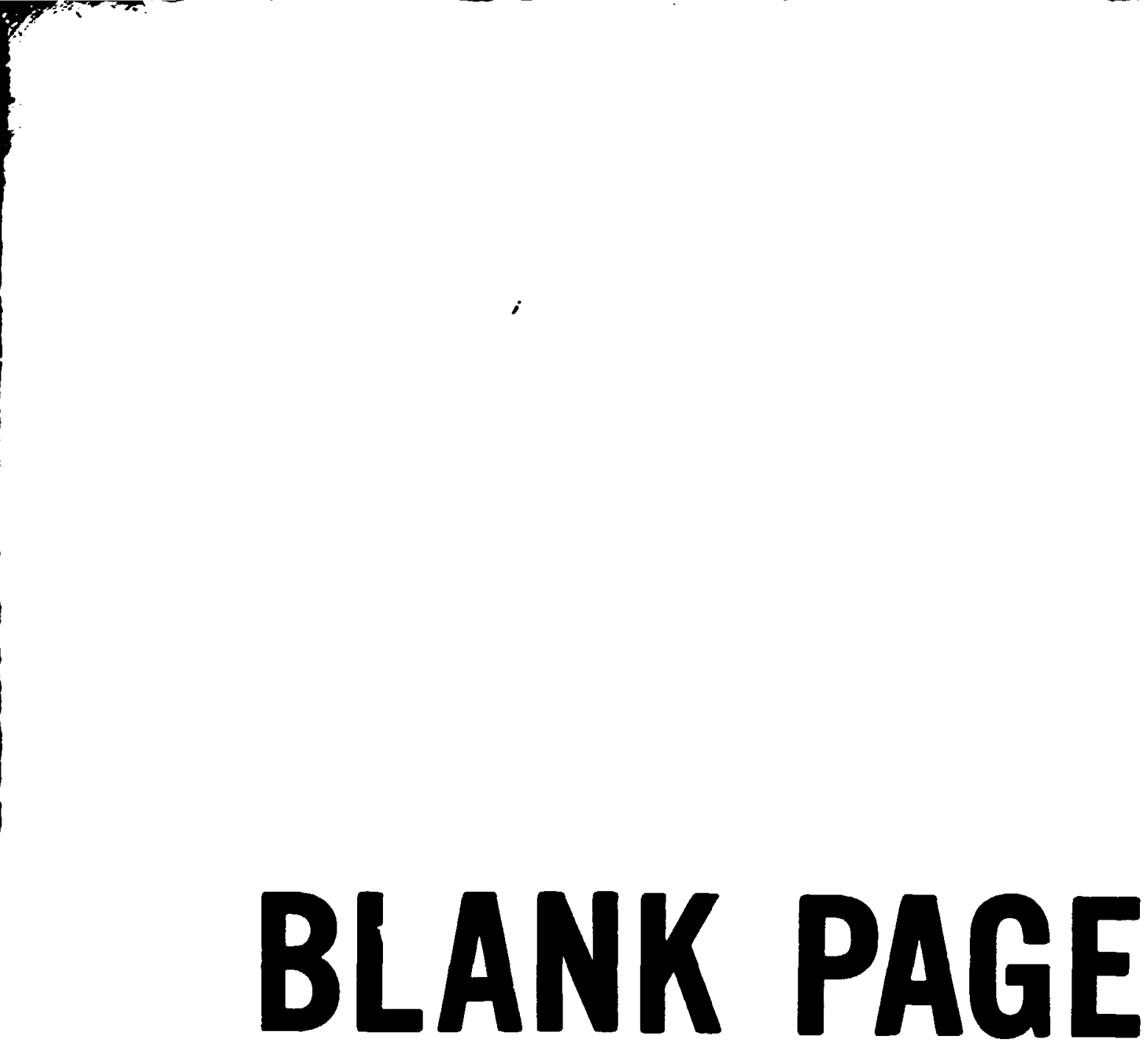




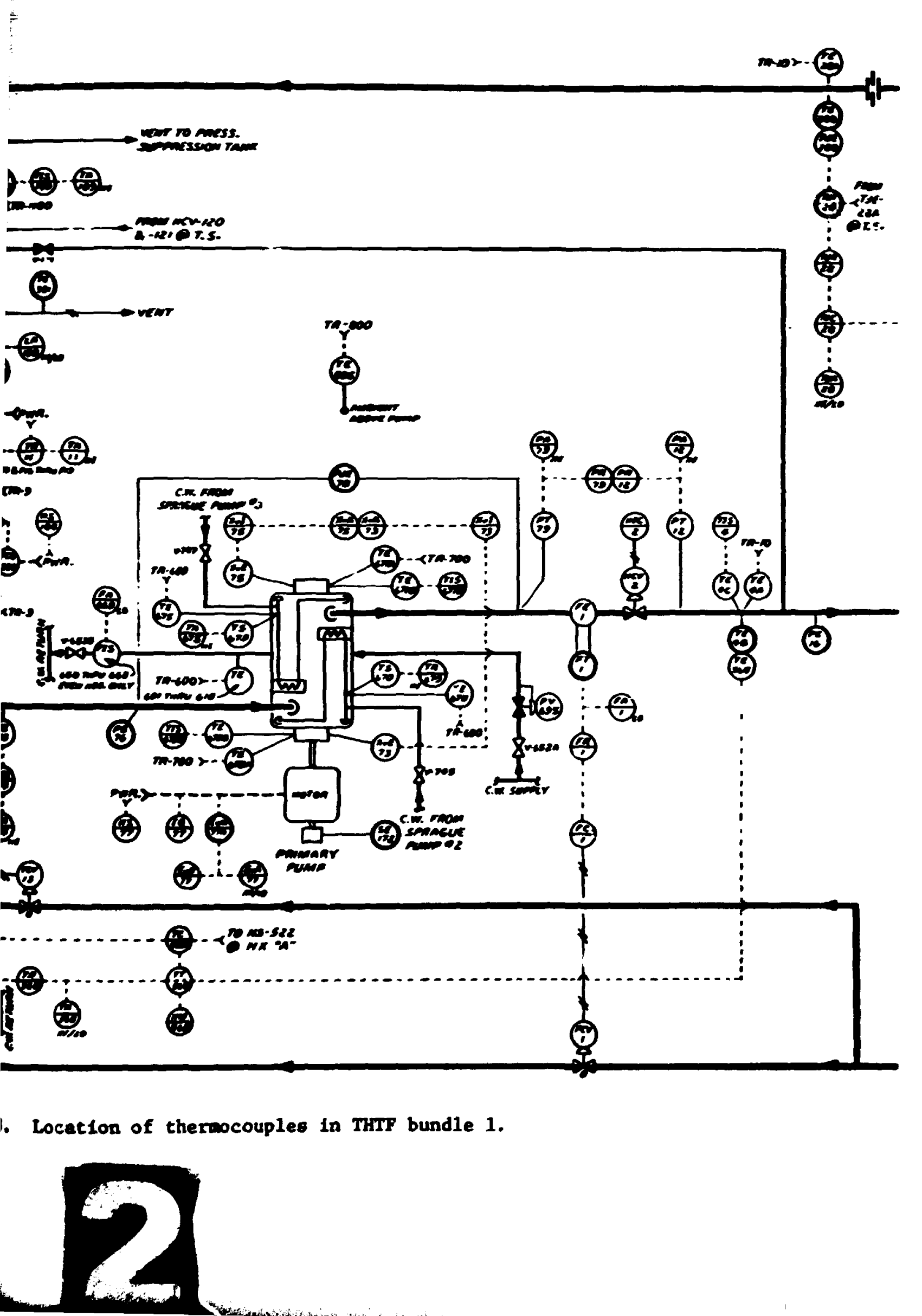




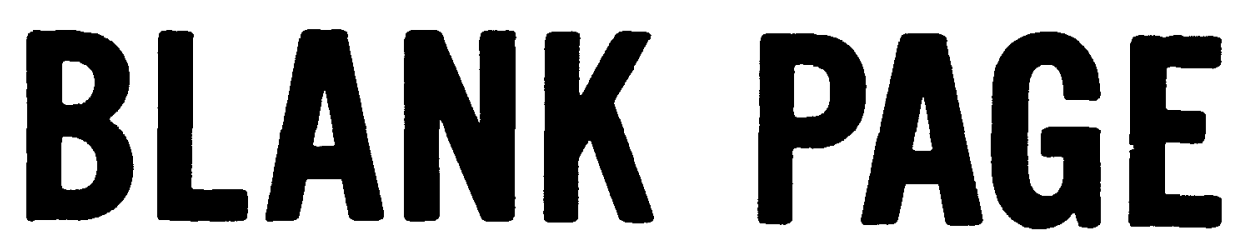




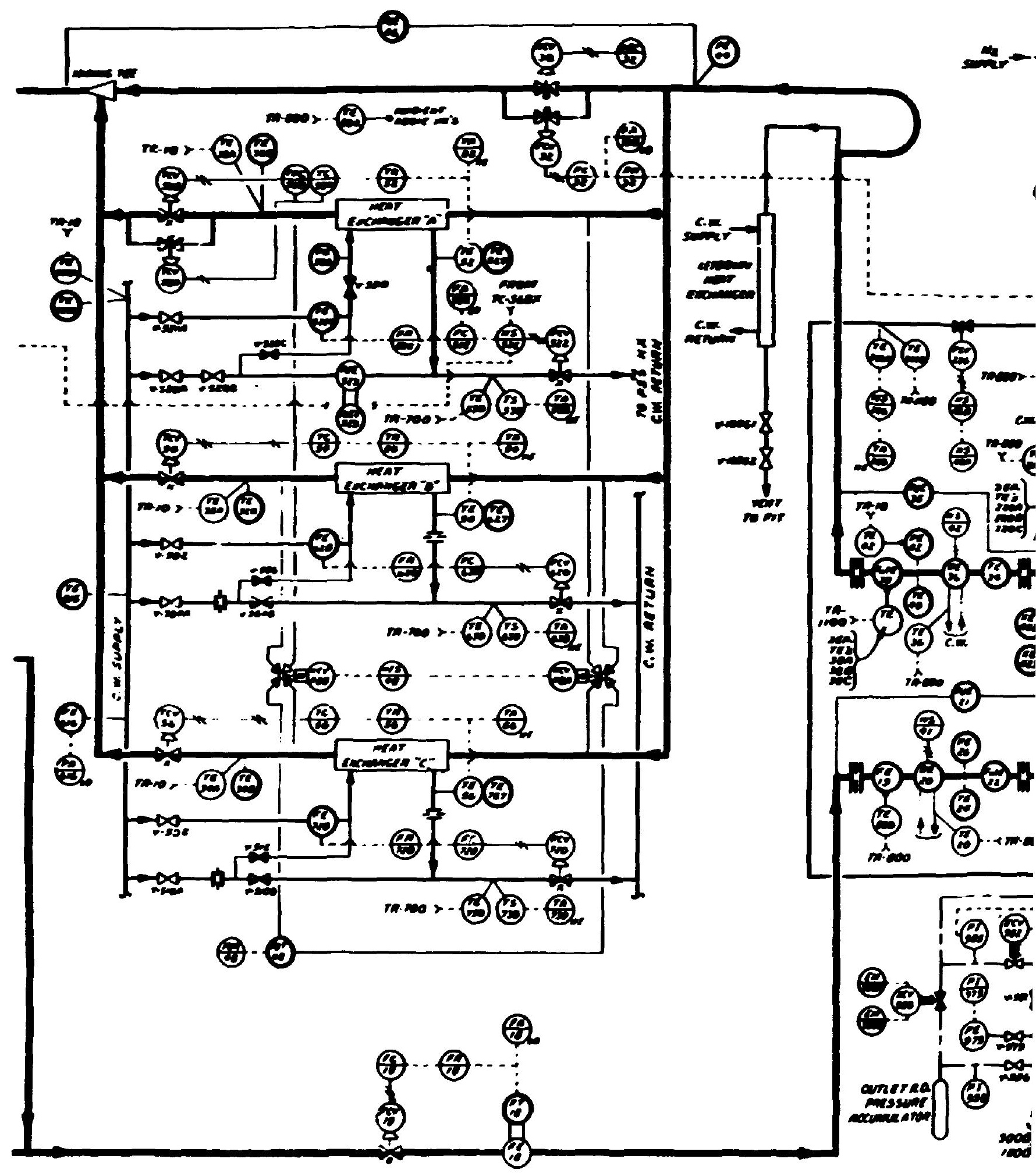




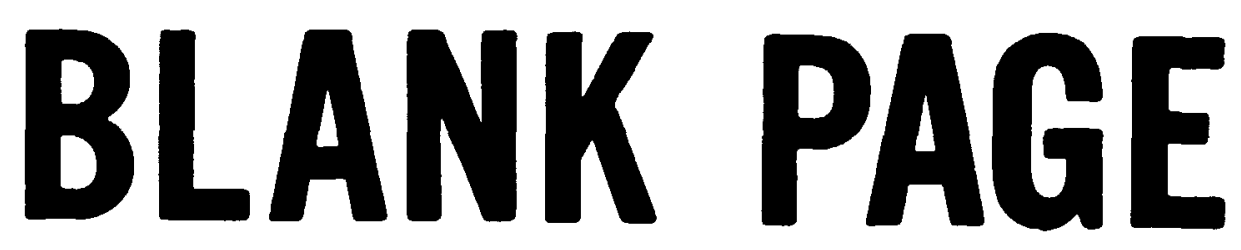




$$
\text { BLANK PAGE }
$$


OTIL-0WG 77-1290

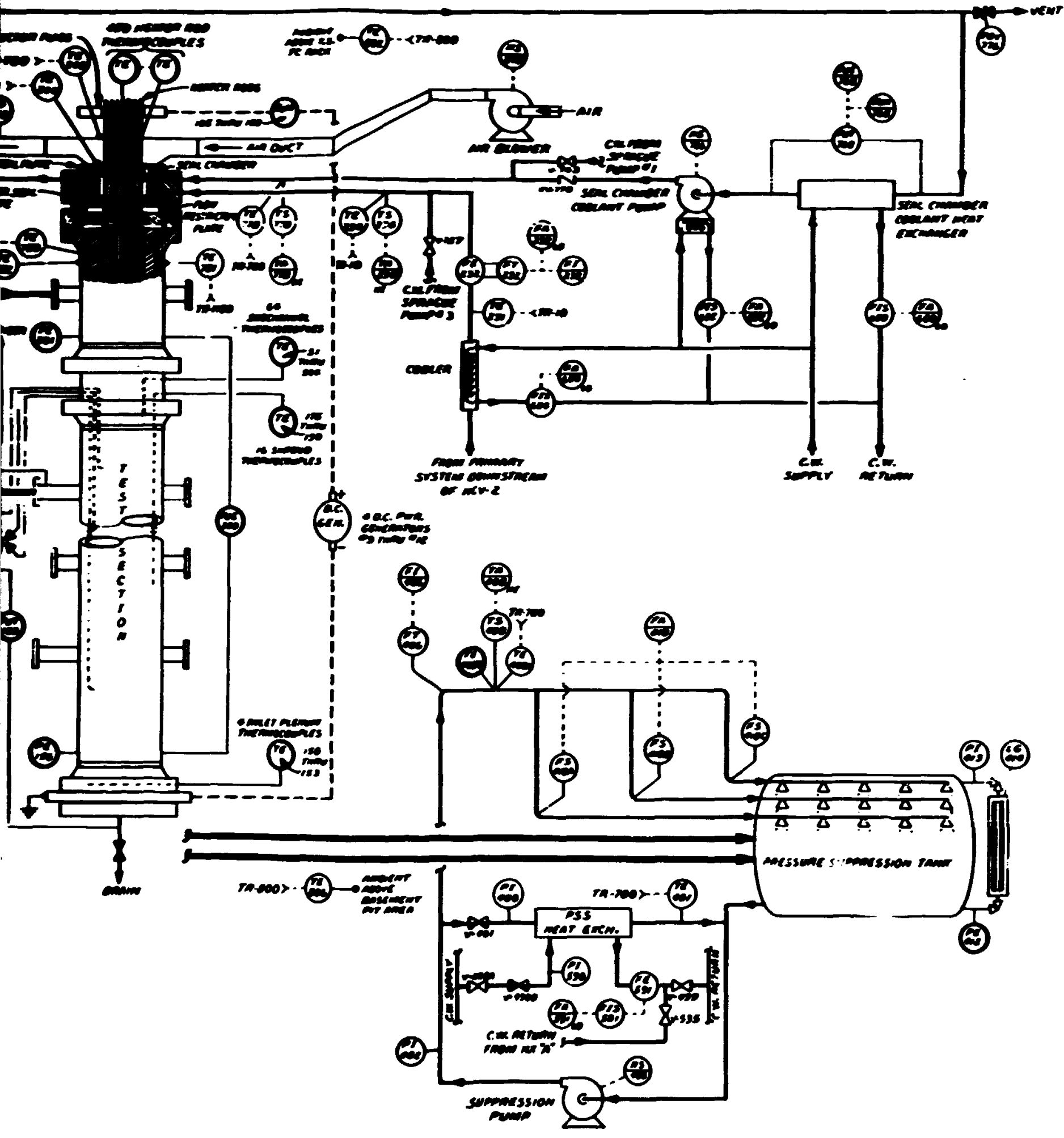


BLANK PAGE 


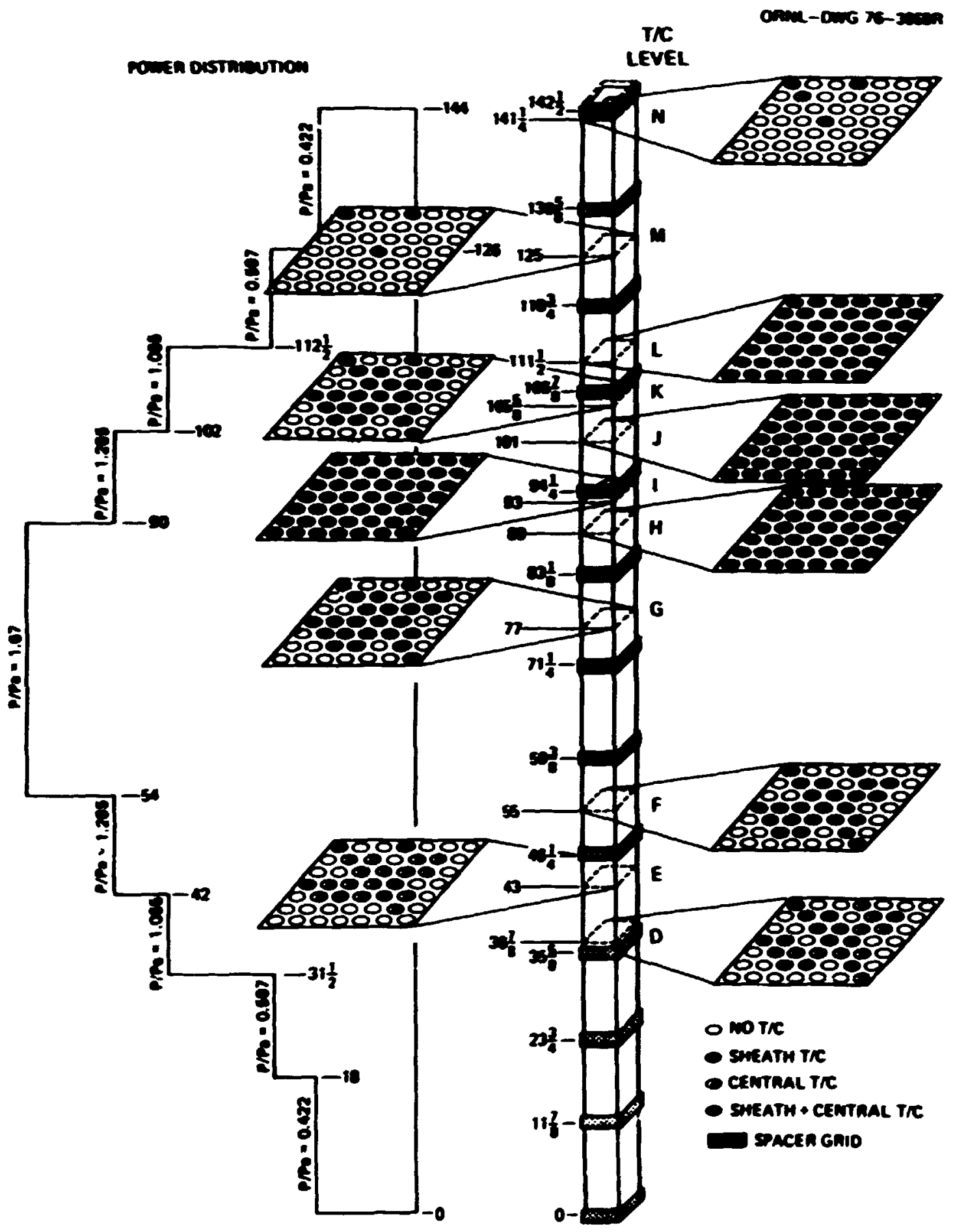

F18. 4. THTP Instrument identification and location. 


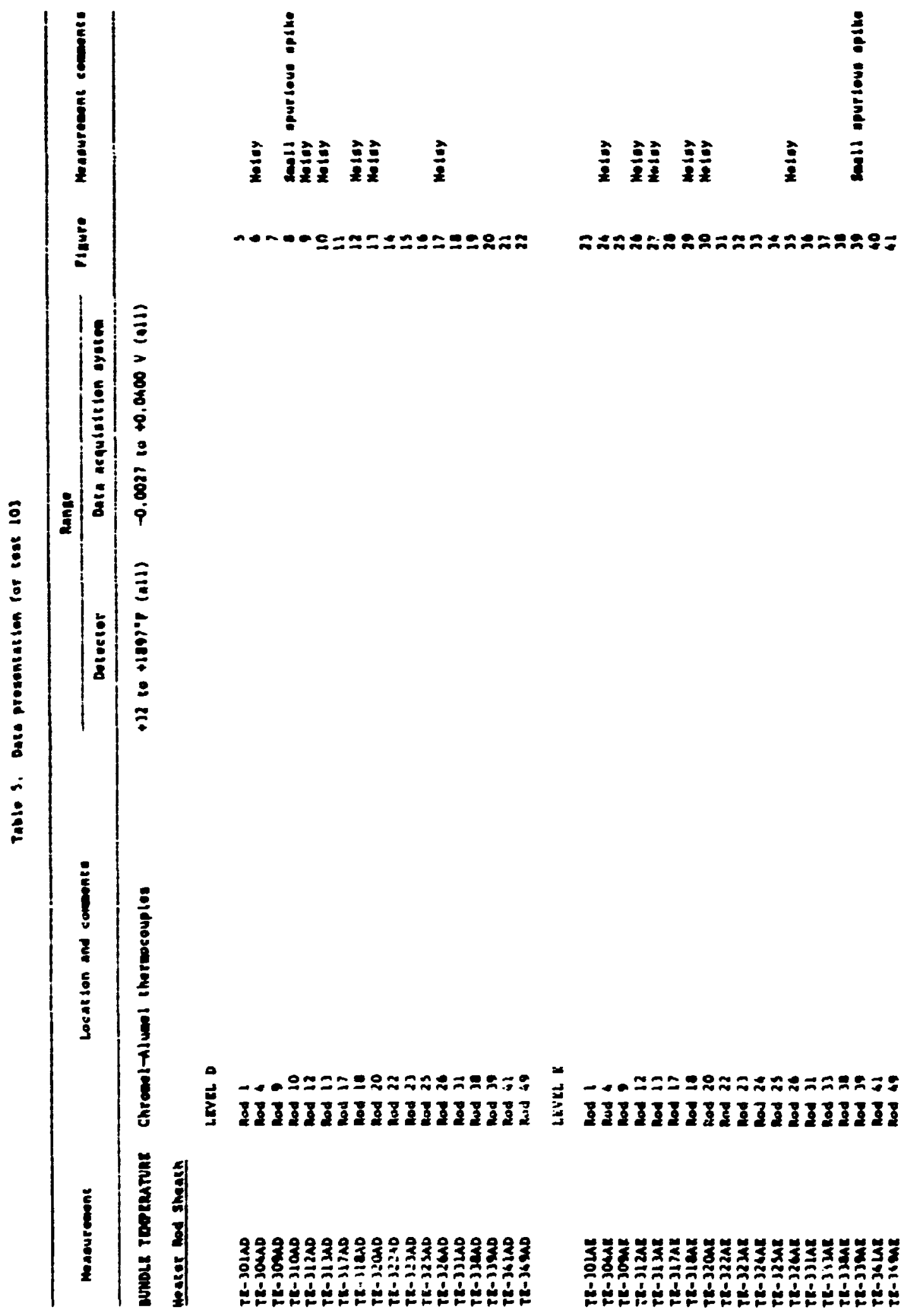


Table 5 (cone lnued)

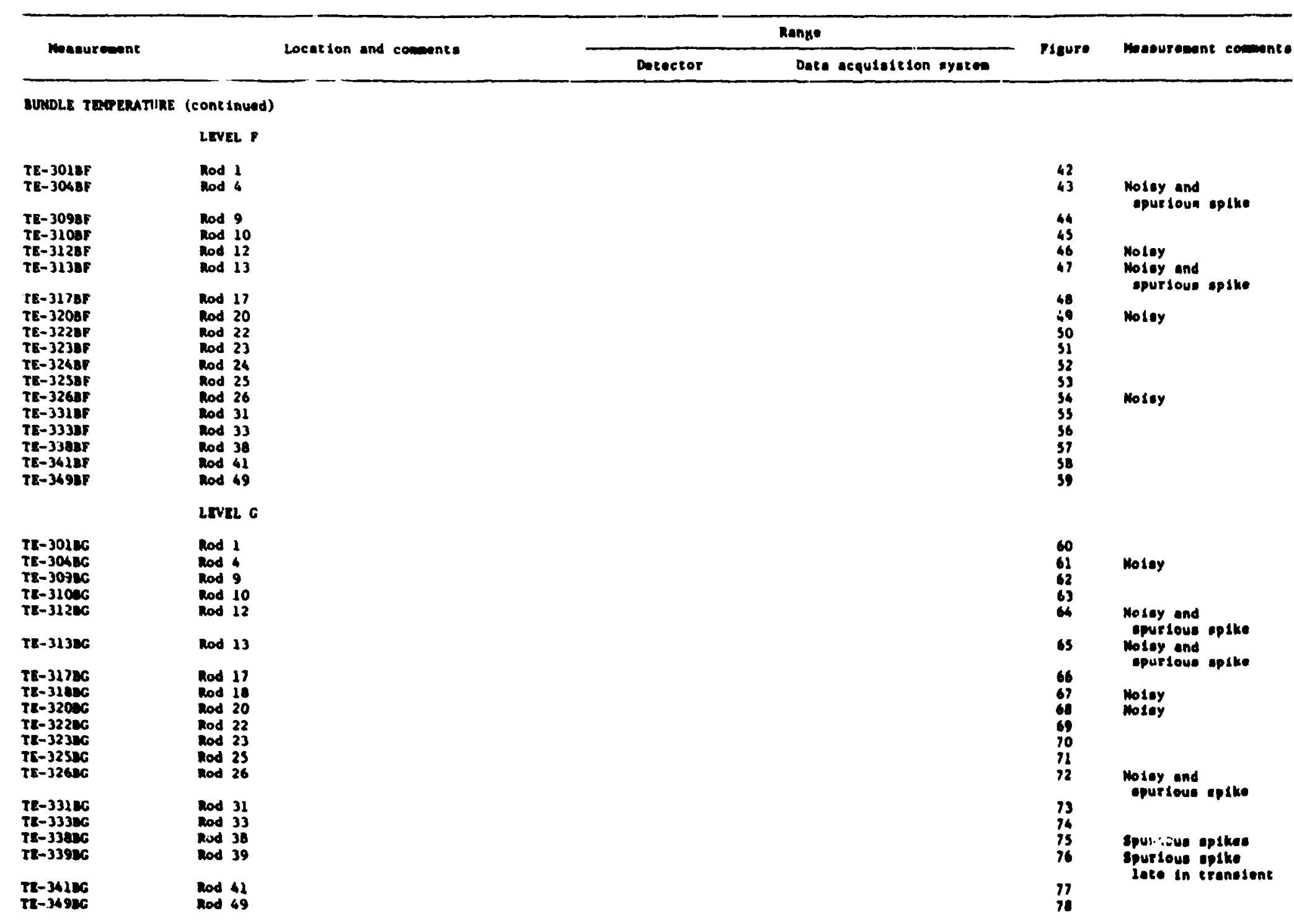




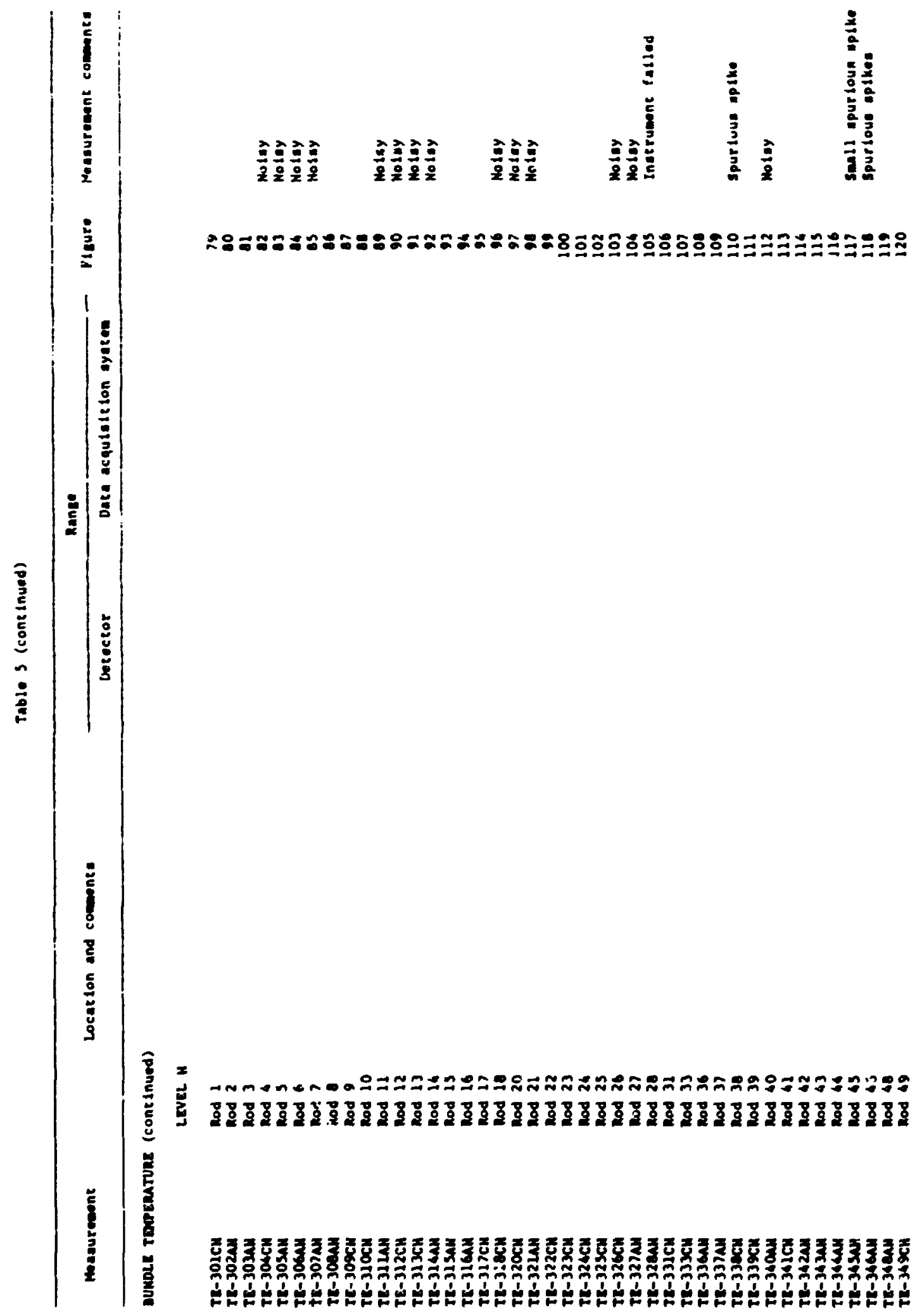


Tablo 5 (continued)

\begin{tabular}{|c|c|c|c|c|c|}
\hline \multirow{2}{*}{ Mesourecent } & \multirow{2}{*}{ Locatson and comente } & \multicolumn{2}{|c|}{ Range } & \multirow{2}{*}{ Pigure } & \multirow{2}{*}{ Macuroesene comenes } \\
\hline & & Datector & Data acquiseltson ayaten & & \\
\hline
\end{tabular}

sudole toormature (continuad)

Lrvat 1

\begin{tabular}{|c|c|}
\hline 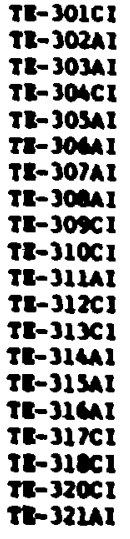 & 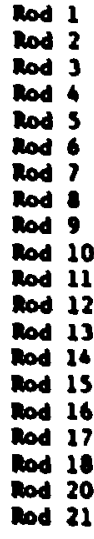 \\
\hline 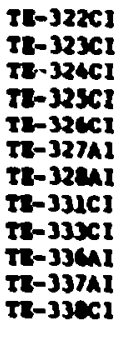 & 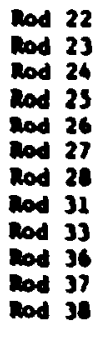 \\
\hline$T[-339 x 1$ & 39 \\
\hline $\begin{array}{l}T E-340 A I \\
T E-341 C I \\
T R-342 A I \\
T z-343 a I\end{array}$ & $\begin{array}{l}\text { nod } 40 \\
\text { nod } 42 \\
\text { nod } 42 \\
\text { nod } 43\end{array}$ \\
\hline
\end{tabular}

Molay

\section{Molny}

Molay and

epup loue apdkes

Molay

Molay

ranll apurtoun

nishos

eanll epur lous

epike lase in

Eranutent

Motey

Spurteue uplke

Lerse apur lous cpikn oarly in 
Tables (contlnued)

\begin{tabular}{|c|c|c|c|c|c|}
\hline \multirow{2}{*}{ Measurement } & \multirow{2}{*}{ Location and coments } & \multicolumn{2}{|c|}{ Range } & \multirow{2}{*}{ Pigure } & \multirow{2}{*}{ Measurement coments } \\
\hline & & Detectar & Data aequilatition gyacom & & \\
\hline
\end{tabular}

OUNDLE TDUPEMTUAE (cone InUEd)

LEVEL I (cont inued)

$\begin{array}{ll}\text { TE-364AI } & \text { Rod } 44 \\ \text { TE-365Al } & \text { Mod } 45 \\ \text { TE-366Al } & \text { Rod } 46 \\ \text { TE-368A1 } & \text { Rod } 48 \\ \text { TE-349C1 } & \text { Mod } 49\end{array}$

LEVEL J

\begin{tabular}{|c|c|}
\hline $\begin{array}{l}\tau E-30 ! D J \\
\tau[-302 C J \\
\tau E-303 C J \\
T E-304 D J\end{array}$ & $\begin{array}{l}\operatorname{Rod} 1 \\
\text { Rod } 2 \\
\text { Rod } 3 \\
\text { Rod } 4\end{array}$ \\
\hline 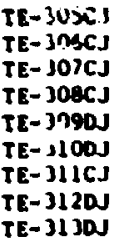 & $\begin{array}{l}\text { Rod } 3 \\
\text { Rod } \\
\text { Rod ? } \\
\text { Rod } \\
\text { Rod } \\
\text { Rod } 10 \\
\text { Rod } 11 \\
\text { Rod } 12 \\
\text { Rod } 13\end{array}$ \\
\hline$T E-314 C]$ & Rod 14 \\
\hline $\begin{array}{l}T E-316 C . J \\
T R-317 D J \\
T E-3190 J \\
T E-3200 J \\
T E-321 C J \\
T E-3220 J \\
T E-323 D \\
T E-326 D \\
T E-323 D \\
T E-3260 J \\
T E-327 C J \\
T E-328 C J\end{array}$ & $\begin{array}{l}\text { Rod } 16 \\
\text { Rod } 17 \\
\text { Rod } 18 \\
\text { Rod } 20 \\
\text { Rod } 21 \\
\text { Rod } 22 \\
\text { Rod } 23 \\
\text { Rod } 26 \\
\text { nod } 25 \\
\text { Rod } 26 \\
\text { hod } 27 \\
\text { Rod } 28\end{array}$ \\
\hline $\begin{array}{l}T E-3310 J \\
T E-3330 J \\
T E-336: J \\
T E-337 C J\end{array}$ & $\begin{array}{l}\operatorname{Rod} 31 \\
\operatorname{Rod} 33 \\
\operatorname{Rod} 36 \\
\operatorname{Rod} 37\end{array}$ \\
\hline
\end{tabular}

159 Spurlous opike

160 spurtous spike

162

63

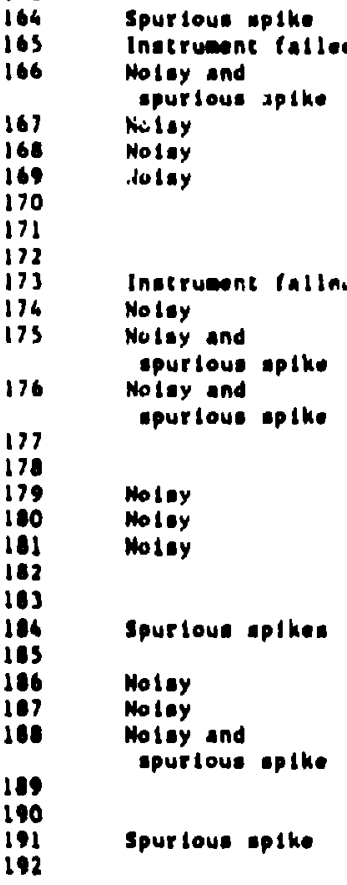


Iabse s (contsavas)

\begin{tabular}{|c|c|c|c|c|c|}
\hline \multirow{2}{*}{ Mosauremont } & \multirow{2}{*}{ Location and comente } & \multicolumn{2}{|c|}{ Renge } & \multirow{2}{*}{ maure } & \multirow{2}{*}{ Meanuromont comonea } \\
\hline & & Datector & Data acqudelts:m ayaton & & \\
\hline \multicolumn{6}{|c|}{ Aunoul tooreutuke (conethued) } \\
\hline & LEVRL J (cont Inued) & & & & \\
\hline 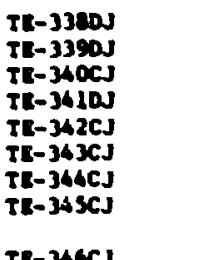 & 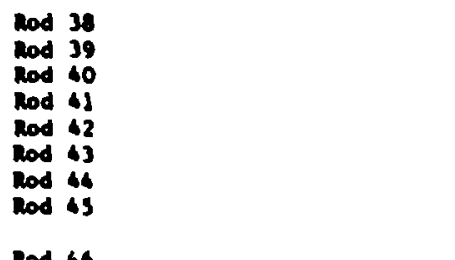 & & & $\begin{array}{l}193 \\
194 \\
195 \\
196 \\
197 \\
190 \\
199 \\
200\end{array}$ & 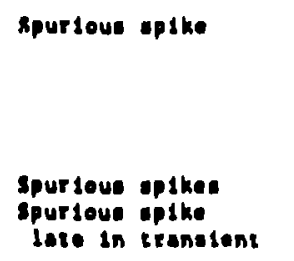 \\
\hline $\begin{array}{l}\text { TE-367CJ } \\
\text { TE-36J }\end{array}$ & $\operatorname{lod} 46$ & & & $\begin{array}{l}200 \\
202\end{array}$ & $\begin{array}{l}\text { seopll epur lous } \\
\text { eplkee arty in } \\
\text { erenasene }\end{array}$ \\
\hline TE-3690] & $\begin{array}{l}\text { Rod ag } \\
\text { LRVEL K }\end{array}$ & & & 203 & \\
\hline $\begin{array}{l}\text { TE-3010K } \\
\text { TE-3040R } \\
\text { TE-3050R }\end{array}$ & $\begin{array}{l}\operatorname{lod} 1 \\
\operatorname{lod} \\
\operatorname{lod}\end{array}$ & & & $\begin{array}{l}204 \\
205 \\
206\end{array}$ & $\begin{array}{l}\text { Mosigy .pine carly } \\
\text { In trenesene }\end{array}$ \\
\hline 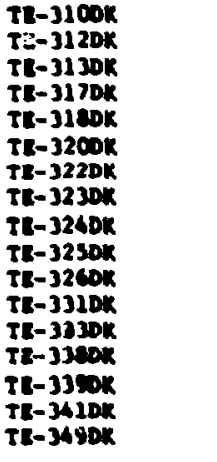 & 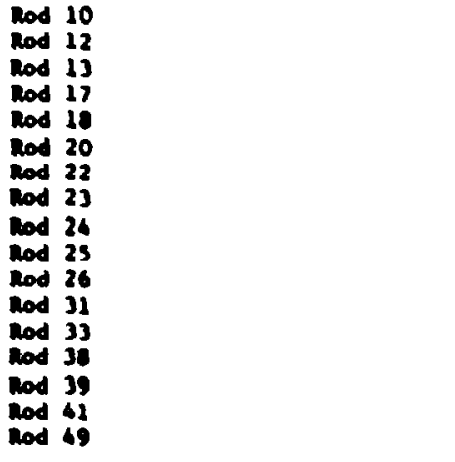 & & & $\begin{array}{l}209 \\
201 \\
209 \\
310 \\
211 \\
212 \\
213 \\
216 \\
213 \\
316 \\
319 \\
211 \\
219 \\
210 \\
221 \\
222 \\
223\end{array}$ & $\begin{array}{l}\text { Moley } \\
\text { Moley } \\
\text { Mosey } \\
\text { Moley }\end{array}$ \\
\hline
\end{tabular}




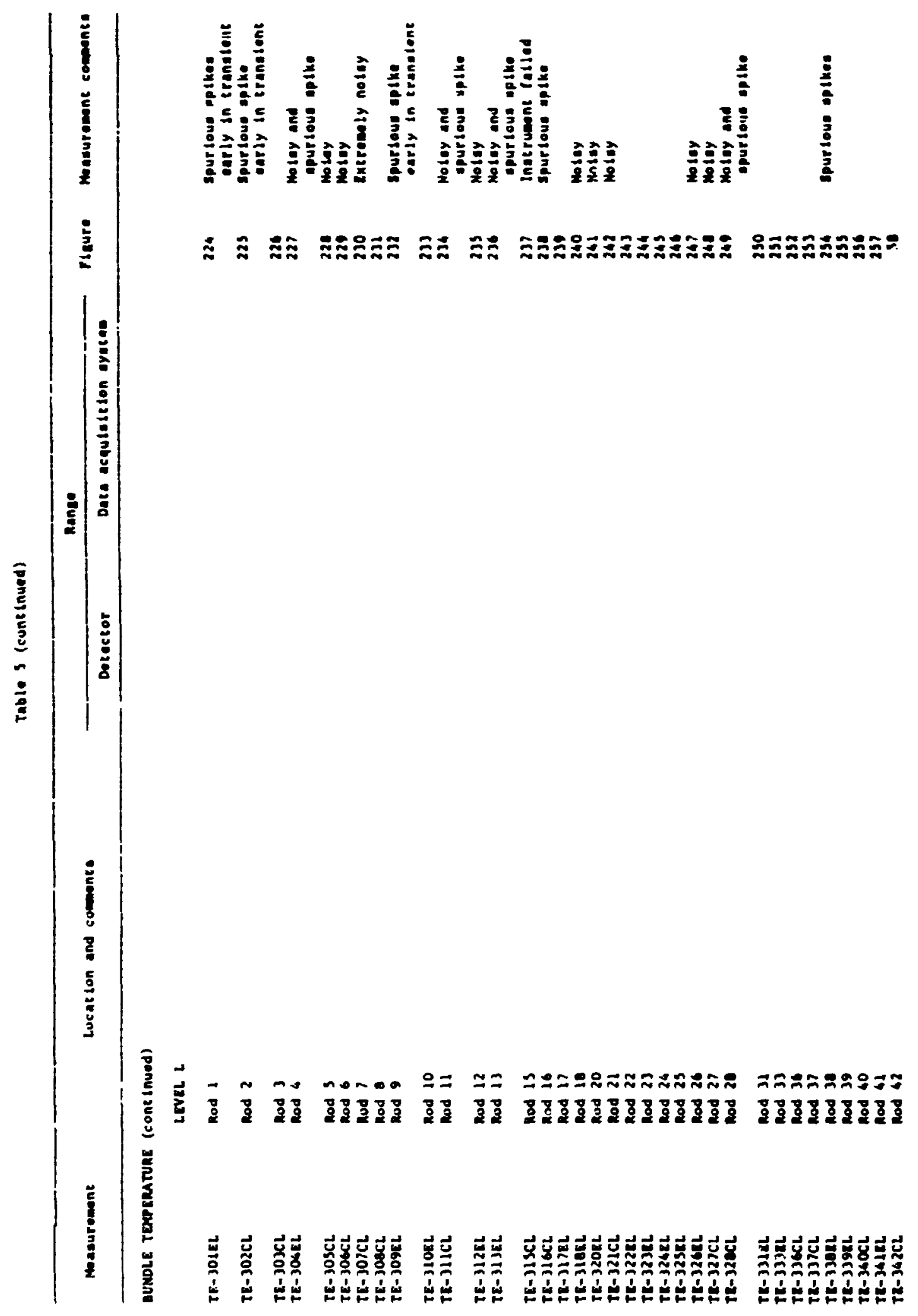


Pable s (cont lnued)

\begin{tabular}{|c|c|c|c|c|c|}
\hline \multirow{2}{*}{ mesuremone } & \multirow{2}{*}{ Locarton and comonts } & \multicolumn{2}{|c|}{ Aenge: } & \multirow{2}{*}{ "igure } & \multirow{2}{*}{ measureaene eamen } \\
\hline & & Dotector & Dute nequblestion aysecon & & \\
\hline \multicolumn{6}{|c|}{ 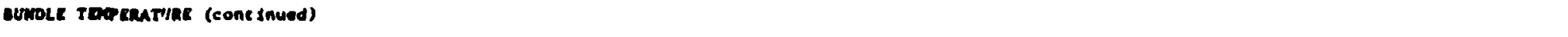 } \\
\hline & LevEL L (conelnued) & & & & \\
\hline \multirow[t]{2}{*}{ 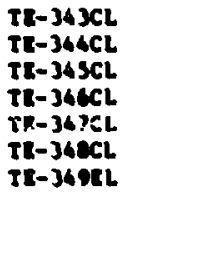 } & $\begin{array}{l}\text { Rod } 43 \\
\text { Rod } 46 \\
\text { Rod } 45 \\
\text { Rod } 46 \\
\text { nod } 47 \\
\text { nod } 40 \\
\text { nod } 49\end{array}$ & & & $\begin{array}{l}239 \\
260 \\
261 \\
362 \\
263 \\
264 \\
263\end{array}$ & $\begin{array}{l}\text { Spurleus seike } \\
\text { spur teue opdkes }\end{array}$ \\
\hline & LEVRL n & & & & \\
\hline \multirow[t]{2}{*}{ 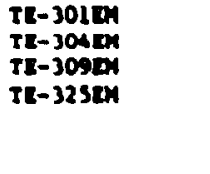 } & $\begin{array}{l}\text { nod ! } \\
\text { nod : } \\
\text { nod as }\end{array}$ & & & $\begin{array}{l}266 \\
261 \\
260 \\
266\end{array}$ & $\begin{array}{l}\text { Moley } \\
\text { spurlous eplko }\end{array}$ \\
\hline & LIVEL N & & & & \\
\hline \multirow[t]{2}{*}{ 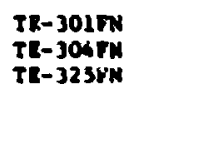 } & $\begin{array}{l}\text { Rod 1 } \\
\text { Rod } \\
\text { Rod } 25\end{array}$ & & & $\begin{array}{l}270 \\
271 \\
212\end{array}$ & Molay \\
\hline & LEVRL 0 & & & & \\
\hline 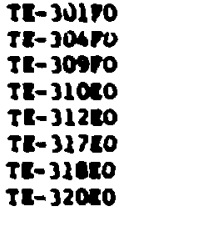 & 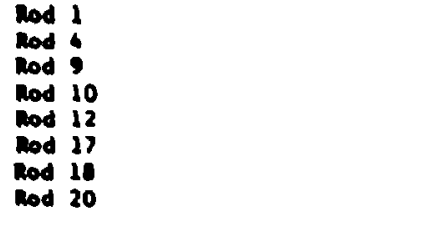 & & & $\begin{array}{l}273 \\
276 \\
278 \\
276 \\
271 \\
276 \\
217 \\
210\end{array}$ & $\begin{array}{l}\text { Mosey } \\
\text { Spurteus apithe } \\
\text { Moloy } \\
\text { Motey } \\
\text { Mosoy and }\end{array}$ \\
\hline 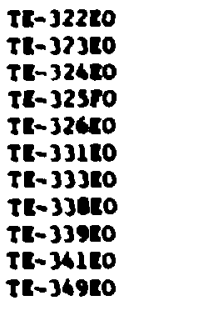 & $\begin{array}{l}\text { Rod } 22 \\
\text { nod } 23 \\
\text { nod } 26 \\
\text { nod } 23 \\
\text { lod } 26 \\
\text { nod } 31 \\
\text { Rod } 33 \\
\text { lod } 30 \\
\text { nod } 39 \\
\text { nod } 41 \\
\text { nod } 49\end{array}$ & & & $\begin{array}{l}201 \\
202 \\
203 \\
204 \\
203 \\
206 \\
201 \\
2010 \\
209 \\
290 \\
291\end{array}$ & 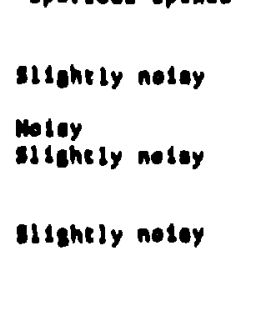 \\
\hline
\end{tabular}


Tables (continued)

\begin{tabular}{|c|c|c|c|c|c|}
\hline \multirow{2}{*}{ Mesuremant } & \multirow{2}{*}{ Loest ton and comente } & \multicolumn{2}{|c|}{ Range } & \multirow{2}{*}{ Disure } & \multirow{2}{*}{ Mesesuremene comenes } \\
\hline & & Detector & sata acquistetion syaten & & \\
\hline \multicolumn{6}{|c|}{ OLNDLE TEORERTURE (continued) } \\
\hline & Level E & & & & \\
\hline $\begin{array}{l}\text { TE-301ME } \\
\text { TR-318mE } \\
\text { TE-322NE }\end{array}$ & $\begin{array}{l}\text { Mod } 1 \\
\text { nod is } \\
\text { Rod } 22 \\
\text { LEVRL P }\end{array}$ & & & $\begin{array}{l}292 \\
291 \\
296\end{array}$ & \\
\hline $\begin{array}{l}\text { TE-30UMP } \\
\text { TE- } 32 \text { JMP }\end{array}$ & $\begin{array}{l}\operatorname{lod} 1 \\
\operatorname{lod} 22\end{array}$ & & & 293 & $\begin{array}{l}\text { Rpur tous epiko } \\
\text { early in eranalene }\end{array}$ \\
\hline & LEVEL G & & & & \\
\hline 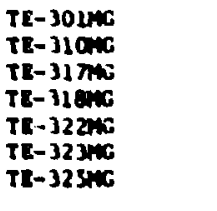 & $\begin{array}{l}\text { Rod } 1 \\
\text { Rod } 10 \\
\text { Rod } 17 \\
\text { Rod } 18 \\
\text { Rod } 22 \\
\text { Rod } 23 \\
\text { Rod } 23\end{array}$ & & & $\begin{array}{l}297 \\
298 \\
290 \\
300 \\
301 \\
302 \\
303\end{array}$ & 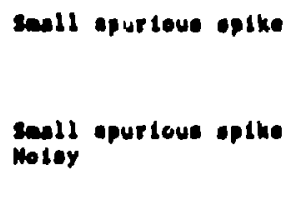 \\
\hline & LEVEL H & & & & \\
\hline 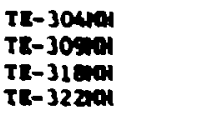 & $\begin{array}{l}\operatorname{Rod} 4 \\
\operatorname{lod} 9 \\
\operatorname{Rod} 18 \\
\operatorname{Rod} 22\end{array}$ & & & $\begin{array}{l}304 \\
j 05 \\
306 \\
307\end{array}$ & \\
\hline & I INVE J & & & & \\
\hline 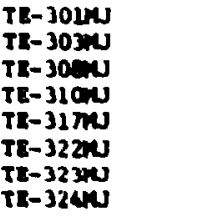 & $\begin{array}{l}\operatorname{Rod} 1 \\
\operatorname{Rod} 3 \\
\operatorname{lod} 8 \\
\operatorname{Rod} 10 \\
\text { nod } 17 \\
\operatorname{lod} 22 \\
\operatorname{Rod} 23 \\
\operatorname{Rod} 26\end{array}$ & & & $\begin{array}{l}300 \\
309 \\
110 \\
311 \\
312 \\
313 \\
314 \\
315\end{array}$ & 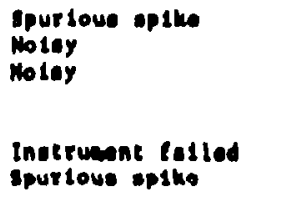 \\
\hline
\end{tabular}


Table I (cone Inued)

\begin{tabular}{|c|c|c|c|c|c|}
\hline \multirow{2}{*}{ Meaturement } & \multirow{2}{*}{ Location and comente } & \multicolumn{2}{|r|}{ Mange } & \multirow{2}{*}{ Pigure } & \multirow{2}{*}{ Mesurtocent comentes } \\
\hline & & Detector & Dete acquilatision oyotom & & \\
\hline \multicolumn{6}{|l|}{$\begin{array}{l}\text { SPool PIEce } \\
\text { IASTminums }\end{array}$} \\
\hline Ievperature & Chromel-Al uend therwocouplea & $+3280+1897^{\circ} \mathrm{T}$ & $-0.0027<0+0.0400 v$ & & \\
\hline $\begin{array}{l}T I-26 \\
T E-172 \\
T E-222 \\
T E-40\end{array}$ & $\begin{array}{l}\text { Horisontel Inlet } \\
\text { Vortleal Inlet } \\
\text { Verticel outlet } \\
\text { Horizontal out lot }\end{array}$ & & & $\begin{array}{l}316 \\
317 \\
318 \\
319\end{array}$ & • \\
\hline Pressure & & O to +3000 peise & $0.0<0+3.0 \mathrm{~V}$ & & \\
\hline $\begin{array}{l}P E-26 \\
P L-176 \\
P E-226 \\
P E-62\end{array}$ & $\begin{array}{l}\text { Horizontel inlet } \\
\text { Vorticel lnlet } \\
\text { Verticel outlet } \\
\text { Wortsontel outlet }\end{array}$ & & & $\begin{array}{l}358 \\
360 \\
363 \\
364\end{array}$ & $\begin{array}{l}\text { Spurlows opikes } \\
\text { spurtous eptkes }\end{array}$ \\
\hline Preseure Drop & & -200 to +200 patd & $-5.010+5.0 \mathrm{~V}$ & & \\
\hline $\begin{array}{l}\text { PDT-21 } \\
\text { PDE-167 } \\
\text { PDE-217 } \\
\text { PDI-3S }\end{array}$ & $\begin{array}{l}\text { Worisontel Inlet } \\
\text { Vorticel Inlet } \\
\text { Verticel outlet } \\
\text { Hordaorted our let }\end{array}$ & & & $\begin{array}{l}309 \\
370 \\
373 \\
374\end{array}$ & 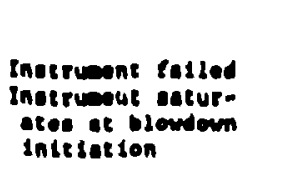 \\
\hline \multicolumn{6}{|l|}{ Doy } \\
\hline $\begin{array}{l}r e-19 \\
T c-166 \\
r c-216 \\
r e-34\end{array}$ & 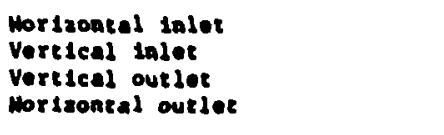 & 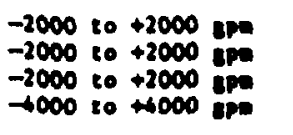 & $\begin{array}{l}-3.010+3.0 \mathrm{~V} \\
-3.010+3.0 \mathrm{v} \\
-3.010+3.0 \mathrm{v} \\
-3.010+3.0 \mathrm{~V}\end{array}$ & $\begin{array}{l}35 s \\
356 \\
357 \\
350\end{array}$ & \\
\hline Veceatin Tlux & & $-250,000$ to $+250,000$ & $-5.0<0+3.0 \mathrm{~V}$ & & \\
\hline 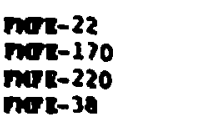 & $\begin{array}{l}\text { Worisontel Inlet } \\
\text { Vertleal Indet } \\
\text { Vortieal outlet } \\
\text { Wordsontal out let }\end{array}$ & & & $\begin{array}{l}306 \\
325 \\
306 \\
301\end{array}$ & Lero entered \\
\hline Euld Danaley & & $0.0+62.626 b_{0} 11 t^{2}$ & 0.0 to $+10.0 \mathrm{~V}$ & & \\
\hline $\begin{array}{l}D L-20 \\
D L=-160 \\
D L-210 \\
D L-36\end{array}$ & $\begin{array}{l}\text { Worisontel ladet } \\
\text { Vertlcal Inlet } \\
\text { Veretcel outlet } \\
\text { Wordzontel out det }\end{array}$ & & & $\begin{array}{l}661 \\
662 \\
663 \\
666\end{array}$ & \\
\hline
\end{tabular}


Tables (conelnued)

\begin{tabular}{|c|c|c|c|c|c|}
\hline \multirow{2}{*}{ Mesuresent } & \multirow{2}{*}{ Locat ton and comente } & \multicolumn{2}{|r|}{ Range } & \multirow{2}{*}{ Figure } & \multirow{2}{*}{ Messurement comone } \\
\hline & & Detector & Dace acquitetetion eyateon & & \\
\hline $\begin{array}{l}\text { Test sEction } \\
\text { TEoranturs }\end{array}$ & Chromel-Alumel elwermecouples & $+32 \cot +1897 \cdot 1$ & $-0.0021<0+0.0400 v$ & & \\
\hline \multicolumn{6}{|l|}{ Dadle shroud } \\
\hline $\begin{array}{l}\mathrm{T}-173 \\
\mathrm{TR}-176 \\
\mathrm{TE}-171 \\
\mathrm{~T}-170\end{array}$ & $\begin{array}{l}0.162 L / L m x \\
0.162 L / L w x \\
0.162 L / L w x \\
0.162 L / L w x\end{array}$ & & & $\begin{array}{l}123 \\
326 \\
327 \\
326\end{array}$ & 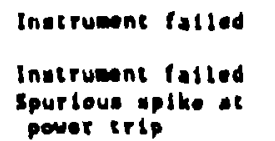 \\
\hline TE-179 & $0.3802 / L m x$ & & & 129 & $\begin{array}{l}\text { Lurse epithe at } \\
\text { power ertp }\end{array}$ \\
\hline TE-180 & $0.3001 / \mathrm{Lux}$ & & & 330 & $\begin{array}{l}\text { Larse ep ike ot } \\
\text { power ertp }\end{array}$ \\
\hline TE-181 & $0.39 a$ / 2 wax & & & 331 & $\begin{array}{l}\text { Lerse apilko at } \\
\text { puwer erip }\end{array}$ \\
\hline $7 t-102$ & $0.3006 / \operatorname{Lrax}$ & & & 332 & $\begin{array}{l}\text { Larse epito at } \\
\text { pewer erip }\end{array}$ \\
\hline $78-183$ & $0.6336 / \mathrm{Lux}$ & & & 333 & $\begin{array}{l}\text { Lorge eptke at } \\
\text { power trip }\end{array}$ \\
\hline TI-1e4 & $0.6332 / L 4 a x$ & & & 334 & $\begin{array}{l}\text { Larke aplke at } \\
\text { poter ertp }\end{array}$ \\
\hline TI-185 & $0.6336 / \mathrm{lmax}$ & & & 335 & $\begin{array}{l}\text { Lorge no iko nt } \\
\text { power trtp }\end{array}$ \\
\hline rt-186 & $0.633 \mathrm{~L} / \mathrm{LWX}$ & & & 336 & $\begin{array}{l}\text { Larea oplike at } \\
\text { power irtp }\end{array}$ \\
\hline$T[-187$ & 0.87 sL/Lunx & & & 337 & $\begin{array}{l}\text { Lorge epike at } \\
\text { pover ertp }\end{array}$ \\
\hline TI-189 & $0 . \operatorname{ersl} / \mathrm{max}$ & & & 338 & $\begin{array}{l}\text { Lurse epthe at } \\
\text { power trip }\end{array}$ \\
\hline$T E-190$ & 0.87 SL/LWNX & & & 339 & $\begin{array}{l}\text { large epithe at } \\
\text { power erlp }\end{array}$ \\
\hline \multicolumn{6}{|c|}{ Medle Subchanael } \\
\hline 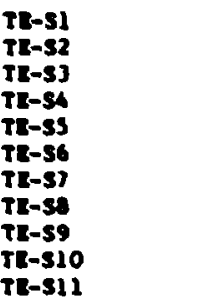 & $\begin{array}{l}\text { Subchanael } 1 \\
\text { Subchaanel } \\
\text { Subchaanel } \\
\text { Subchanael } \\
\text { Subchanael } \\
\text { Subchanael } \\
\text { Subchanael } \\
\text { Subchunael } \\
\text { Subchanael } \\
\text { Subchannel } 10 \\
\text { Subchannel il }\end{array}$ & & & $\begin{array}{l}473 \\
474 \\
475 \\
476 \\
477 \\
418 \\
479 \\
400 \\
481 \\
462 \\
481\end{array}$ & 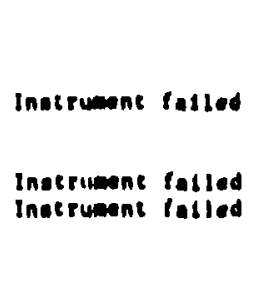 \\
\hline
\end{tabular}


Tiblo S (cont inued)

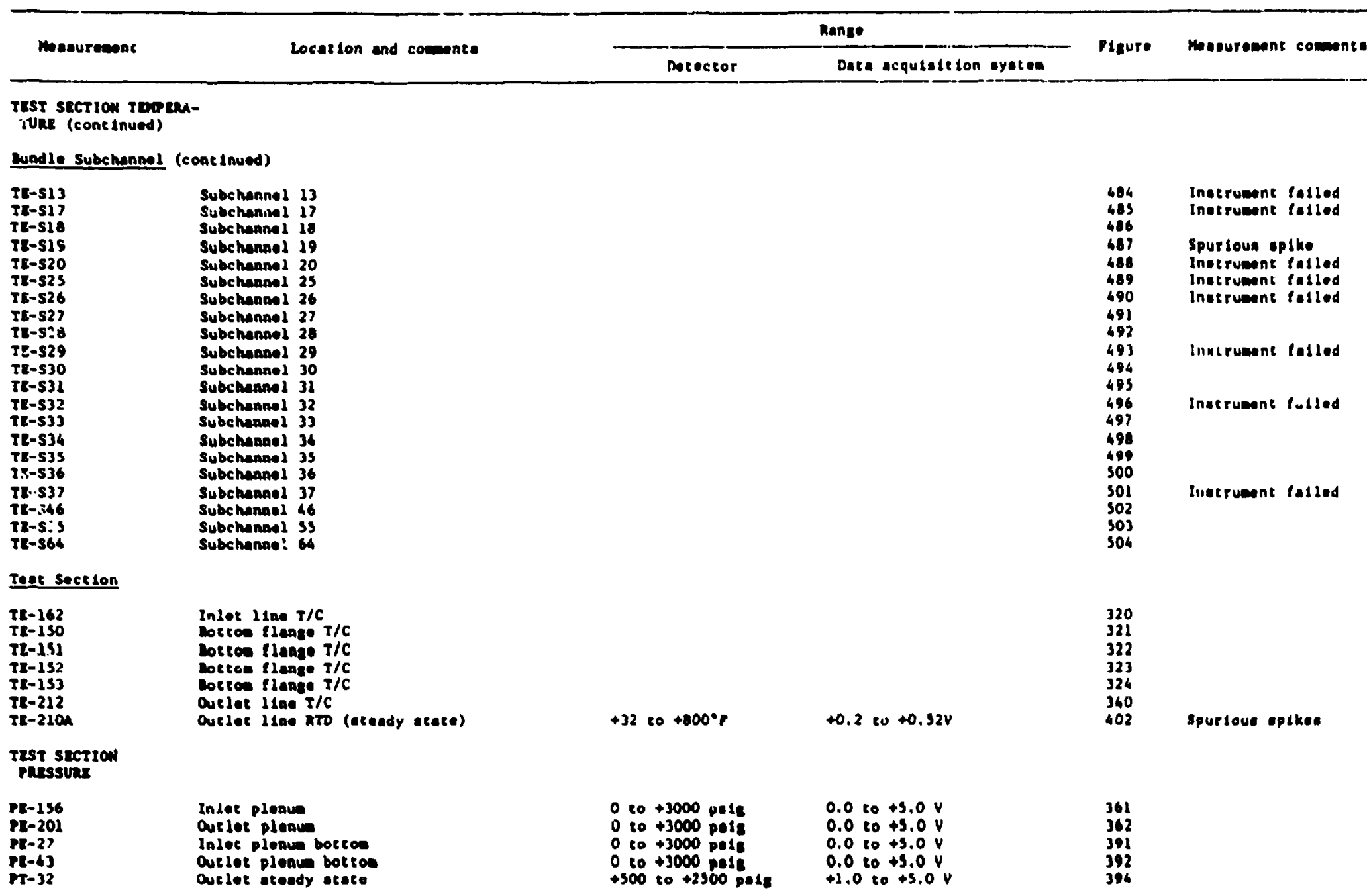




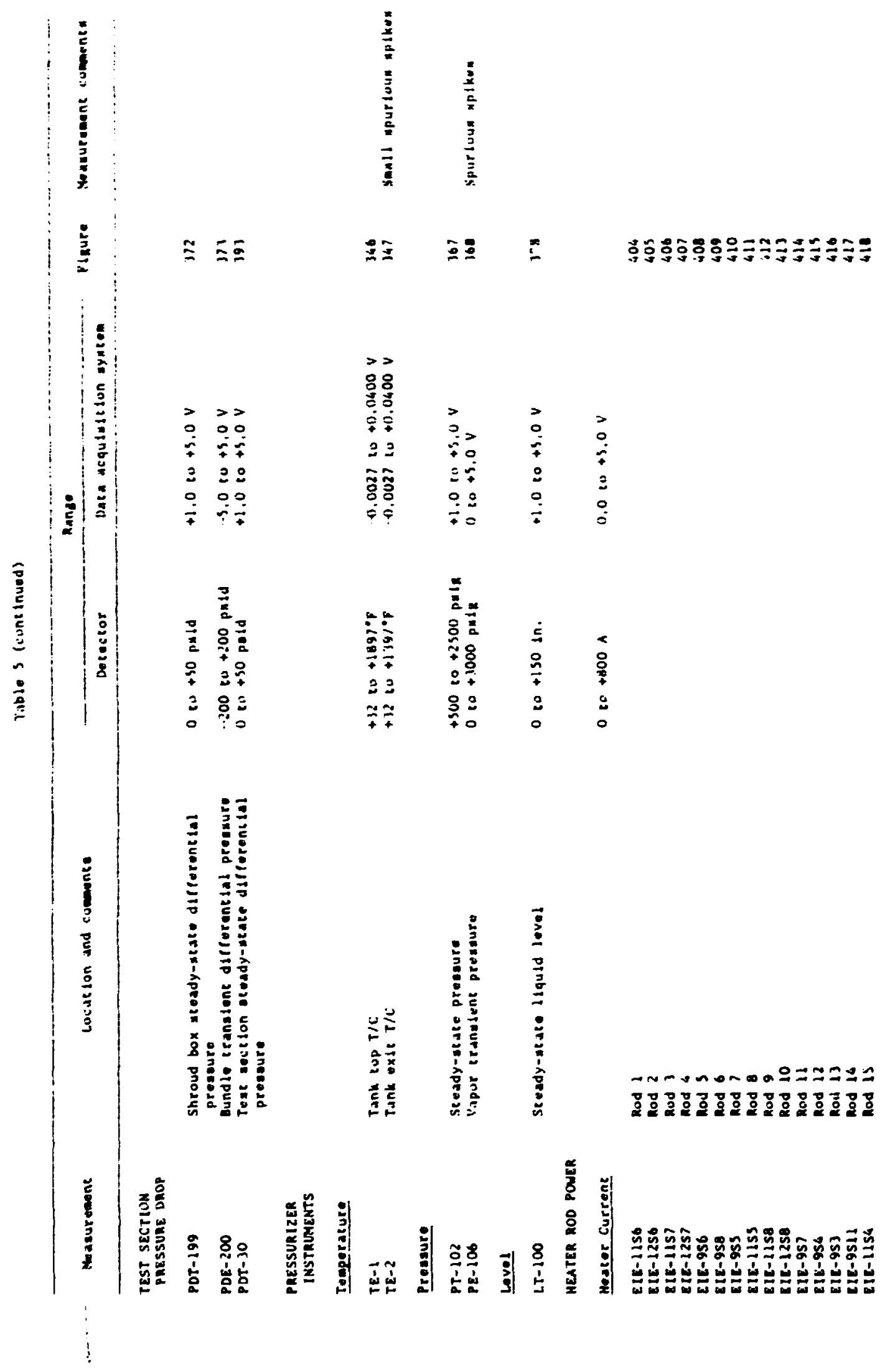


Tables (cone inued)

\begin{tabular}{|c|c|c|c|c|c|}
\hline \multirow{2}{*}{ masuraeone } & \multirow{2}{*}{ Locatton and coments } & \multicolumn{2}{|c|}{ Range } & \multirow{2}{*}{ Mgute } & \multirow{2}{*}{ Monsurezent } \\
\hline & & Detector & Data acquitalition myateon & & \\
\hline
\end{tabular}

MeATER ROD POWER (cone Inved)

Mescer Current (cone lnued)

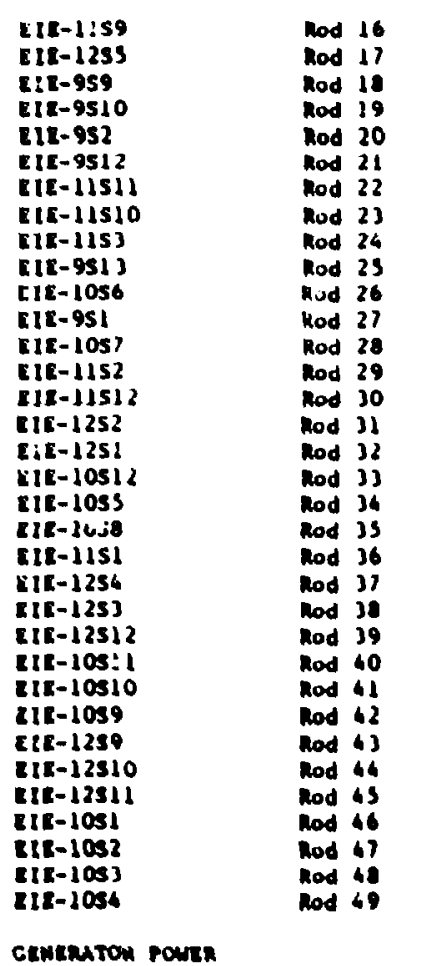

Gewiston pours

Generaker currene

R1E-9
RiR-10

429
420
421
422
423
424
425
426
427
428
429
410
431
432
431
434
433
436
417
434
439
440
441
442
441
444
463
446
447
446
449
490
451
492

Ganerater Pe currone

0 to $+10,000 A$

$0.0+0+2.5 \mathrm{~V}$

433 

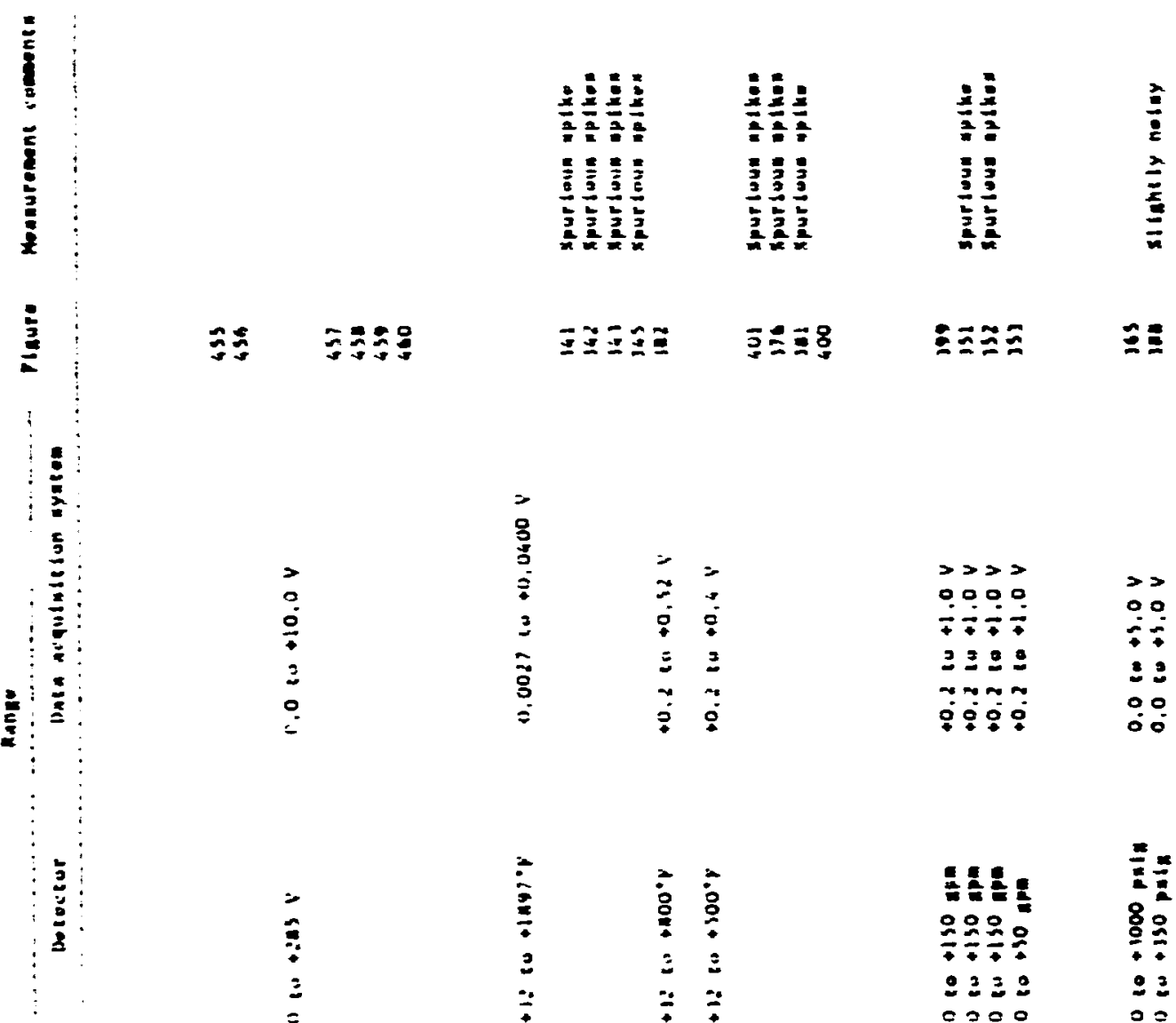

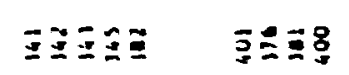

シ三三ミ

EE
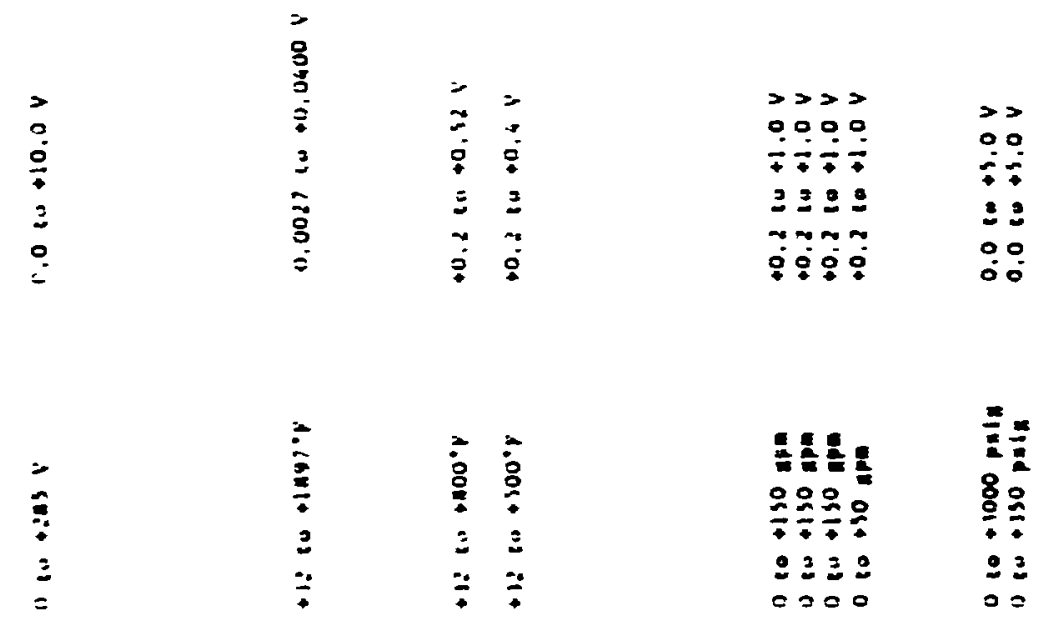

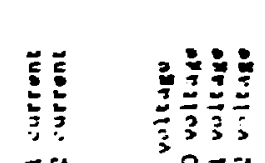
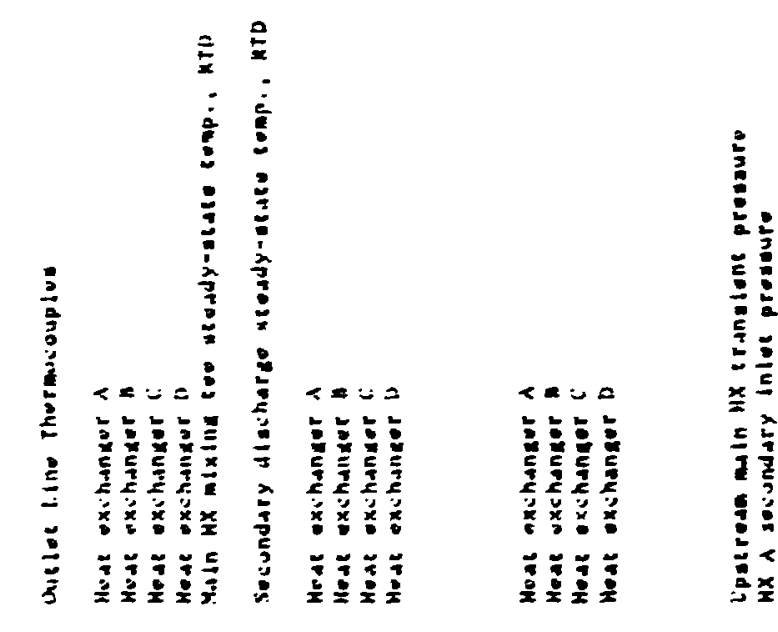

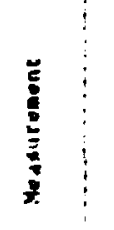

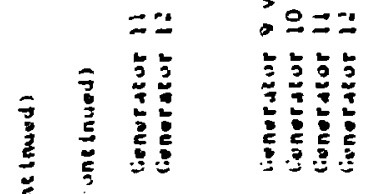

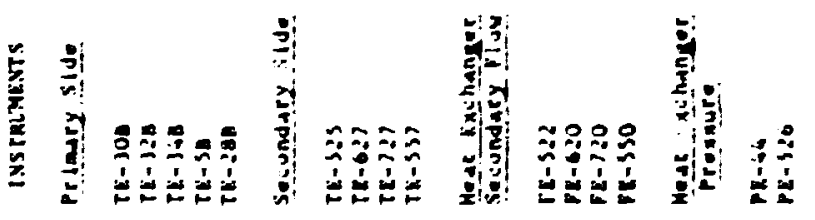


Table 5 (cone inued)

\begin{tabular}{|c|c|c|c|c|c|}
\hline \multirow{2}{*}{ Measurement } & \multirow{2}{*}{ Location and coments } & \multicolumn{2}{|r|}{ Rance } & \multirow{2}{*}{ Figure } & \multirow{2}{*}{ Mensurenent coments } \\
\hline & & Detector & Data arquilation syatem & & \\
\hline \multicolumn{6}{|c|}{$\begin{array}{l}\text { HEAT EXCLUNGER (cont InUed) } \\
\text { INSTRUMENTS }\end{array}$} \\
\hline \multicolumn{6}{|l|}{ Pressure Drop } \\
\hline $\begin{array}{l}\text { PDT- } 48 \\
\text { PDE : } 6\end{array}$ & $\begin{array}{l}\text { Maln } H X \text { steady-gtate pressure drop } \\
\text { Maln HX bypass tranglent pressure drop }\end{array}$ & $\begin{array}{l}0 \text { co }+24 \text { psidd } \\
-200 \text { co }+200 \text { paid }\end{array}$ & $\begin{array}{l}+1.0 \mathrm{tu}+5.0 \mathrm{~V} \\
-9.0 \mathrm{co+5.0 \textrm {V }}\end{array}$ & $\begin{array}{l}317 \\
383\end{array}$ & $\begin{array}{l}\text { Instrument catur- } \\
\text { ecea at blowdown } \\
\text { instiat ton }\end{array}$ \\
\hline \multicolumn{6}{|c|}{$\begin{array}{l}\text { PRIMARY PLEP INSTR!:- } \\
\text { MENTS }\end{array}$} \\
\hline $\begin{array}{l}\text { PE-1A } \\
\text { PE-76 } \\
P D E-78 \\
\text { SE }-72 \\
\text { TE-48 } \\
\text { PE-16 }\end{array}$ & $\begin{array}{l}\text { Primary side pump flow } \\
\text { Pump suction transient preasure } \\
\text { Primary pump transient pressure drop } \\
\text { Primary puap speed } \\
\text { dase primar: steady-state cemp. RTD } \\
\text { Dommetream HCV-2 cransient pregsure }\end{array}$ & 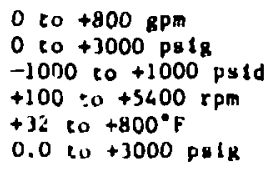 & $\begin{array}{l}+1.0+0+5.0 v \\
0<0+5.0 v \\
-5.020+5.0 v \\
0<0+5.0 v \\
+0.210+0.52 v \\
0.010+5.0 v\end{array}$ & $\begin{array}{l}354 \\
366 \\
378 \\
395 \\
350 \\
390\end{array}$ & $\begin{array}{l}\text { Spurious apikes } \\
\text { Spurloue opikes } \\
\text { Spurlouk opikes }\end{array}$ \\
\hline \multicolumn{6}{|c|}{$\begin{array}{l}\text { PRESSURE SUPPRESSION } \\
\text { SYSTEM INSTRLMENTS }\end{array}$} \\
\hline$P E-412$ & $\begin{array}{l}\text { Pressure suppression recelvar transient } \\
\text { pressure }\end{array}$ & 0 to +200 psig & $0<0+9.0 \mathrm{~V}$ & 369 & \\
\hline $\begin{array}{l}P E-425 \\
P E-427 \\
\text { TE-29 } \\
-E-45\end{array}$ & 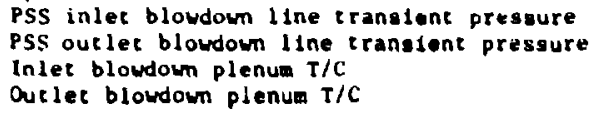 & 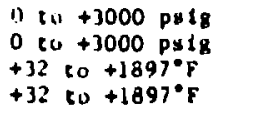 & $\begin{array}{l}0<0+3.0 v \\
0<0+5.0 v \\
-0.0027<0+0.0400 v \\
-0.0027 \text { to +0.0400 v }\end{array}$ & $\begin{array}{l}379 \\
380 \\
348 \\
349\end{array}$ & $\begin{array}{l}\text { Spuriuus eplkes } \\
\text { Spurlous apikes }\end{array}$ \\
\hline \multicolumn{6}{|l|}{$\begin{array}{l}\text { DEMINERAL IZED } \\
\text { WATER SYSTEM }\end{array}$} \\
\hline $\begin{array}{l}\text { TE-520B } \\
\text { TE-61S }\end{array}$ & $\begin{array}{l}\text { RBT } 4 \text { In, deminerallzed water header } T / C \\
\text { Deminerallzed vater } 6-1 n \text {. heuder steady- } \\
\text { stace tomp. RTD }\end{array}$ & $\begin{array}{l}+3280+189)^{\circ} \mathrm{F} \\
+32 \cdot \mathrm{F} 80+300^{\circ} \mathrm{F}\end{array}$ & $\begin{array}{l}-0.0027<0+0.0400 v \\
+0.2 \text { to }+0.4 v\end{array}$ & $\begin{array}{l}344 \\
371\end{array}$ & Inrge spurious splkes \\
\hline \multicolumn{6}{|c|}{$\begin{array}{l}\text { GENERAL INSTRURENTA- } \\
\text { TATION (ELECTRICAL) }\end{array}$} \\
\hline \multicolumn{6}{|c|}{ Breakuilre Detectors } \\
\hline $\begin{array}{l}x 4-60001 \\
x+40000\end{array}$ & $\begin{array}{l}\text { Inlee break } \\
\text { outlet break }\end{array}$ & $\begin{array}{l}0.010+5.0 v \\
0.0 \text { to }+5.0 v\end{array}$ & $\begin{array}{l}0.0 \mathrm{en}+5.0 \mathrm{~V} \\
0.0 \mathrm{co}+5.0 \mathrm{~V}\end{array}$ & $\begin{array}{l}396 \\
397\end{array}$ & \\
\hline
\end{tabular}




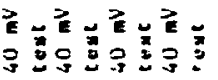

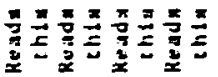

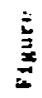

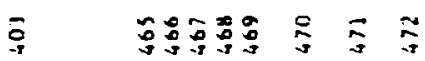

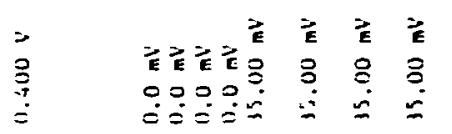

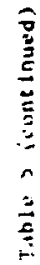

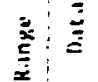

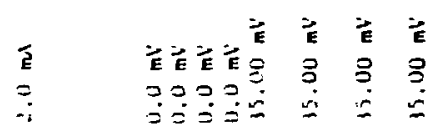

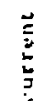

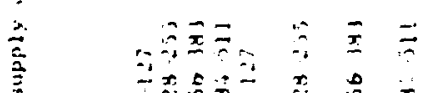

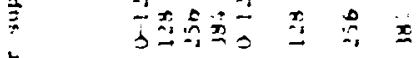

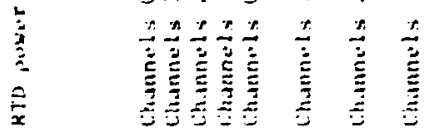

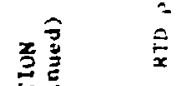

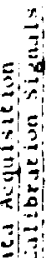

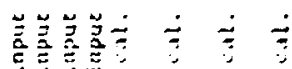

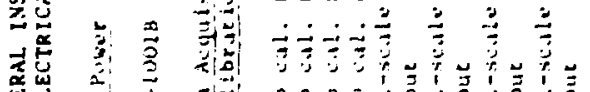

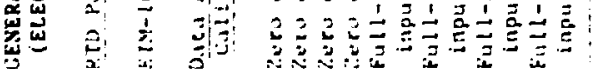

